\title{
A guide for urogynecologic patient care utilizing telemedicine during the COVID-19 pandemic: review of existing evidence
}

\author{
Cara L. Grimes ${ }^{1}$ - Ethan M. Balk ${ }^{2}$ - Catrina C. Crisp ${ }^{3}$ - Danielle D. Antosh ${ }^{4} \cdot$ Miles Murphy $^{5} \cdot$ Gabriela E. Halder $^{6}$. \\ Peter C. Jeppson ${ }^{7}$. Emily E. Weber LeBrun ${ }^{8}$. Sonali Raman ${ }^{9}$. Shunaha Kim-Fine ${ }^{10} \cdot$ Cheryl Iglesia $^{11}$. \\ Alexis A. Dieter ${ }^{12} \cdot$ Ladin Yurteri-Kaplan $^{13} \cdot$ Gaelen Adam $^{2} \cdot$ Kate V. Meriwether $^{7}$
}

Received: 6 April 2020 / Accepted: 7 April 2020 / Published online: 27 April 2020

(C) The International Urogynecological Association 2020

\begin{abstract}
Introduction and hypothesis The COVID-19 pandemic and the desire to "flatten the curve" of transmission have significantly affected the way providers care for patients. Female Pelvic Medicine and Reconstructive Surgeons (FPMRS) must provide high quality of care through remote access such as telemedicine. No clear guidelines exist on the use of telemedicine in FPMRS. Using expedited literature review methodology, we provide guidance regarding management of common outpatient urogynecology scenarios during the pandemic.

Methods We grouped FPMRS conditions into those in which virtual management differs from direct in-person visits and conditions in which treatment would emphasize behavioral and conservative counseling but not deviate from current management paradigms. We conducted expedited literature review on four topics (telemedicine in FPMRS, pessary management, urinary tract infections, urinary retention) and addressed four other topics (urinary incontinence, prolapse, fecal incontinence, defecatory dysfunction) based on existing systematic reviews and guidelines. We further compiled expert consensus regarding management of FPMRS patients in the virtual setting, scenarios when in-person visits are necessary, symptoms that should alert providers, and specific considerations for FPMRS patients with suspected or confirmed COVID-19.

Results Behavioral, medical, and conservative management will be valuable as first-line virtual treatments. Certain situations will require different treatments in the virtual setting while others will require an in-person visit despite the risks of COVID-19 transmission.

Conclusions We have presented guidance for treating FPMRS conditions via telemedicine based on rapid literature review and expert consensus and presented it in a format that can be actively referenced.
\end{abstract}

Cara L. Grimes

caragrimesmd@gmail.com

1 Departments of Obstetrics and Gynecology and Urology, New York Medical College, 19 Bradhurst Avenue, Suite 2700 South Hawthorne, Valhalla, NY 10532, USA

2 Center for Evidence Synthesis in Health, Brown School of Public Health, Brown University, Providence, RI, USA

3 Department of Obstetrics and Gynecology, Division of Female Pelvic Medicine and Reconstructive Surgery, TriHealth, Cincinnati, OH, USA

4 Department of Obstetrics and Gynecology, Division of Urogynecology, Houston Methodist Hospital, Houston, TX, USA

5 Department of Obstetrics and Gynecology, Sidney Kimmel Medical College of Thomas Jefferson University, Philadelphia, PA, USA

6 Department of Women's Health, Dell Medical School, University of Texas Austin, Austin, TX, USA
7 Division of Female Pelvic Medicine and Reconstructive Surgery, Department of Obstetrics and Gynecology, University of New Mexico, Albuquerque, NM, USA

8 Division of Female Pelvic Medicine and Reconstructive Surgery, Department of Obstetrics and Gynecology, University of Florida, Gainesville, FL, USA

9 Department of Women's Health, Female Pelvic Medicine and Reconstructive Surgery, St. Elizabeth Healthcare, Fort Thomas, KY, USA

10 Section of Pelvic Medicine and Reconstructive Surgery, Department of Obstetrics and Gynecology, University of Calgary, Calgary, Alberta, Canada

11 Department of Obstetrics and Gynecology, Georgetown University School of Medicine, Washington, DC, USA

12 Division of Urogynecology and Reconstructive Pelvic Surgery, Department of Obstetrics and Gynecology, University of North Carolina at Chapel Hill, Chapel Hill, NC, USA

13 Department of Obstetrics and Gynecology, Columbia University Irving Medical Center, New York, NY, USA 
Keywords COVID-19 $\cdot$ Urogynecology $\cdot$ FPMRS $\cdot$ Telemedicine $\cdot$ Systematic review $\cdot$ Pandemic $\cdot$ Virtual visit

\section{Introduction}

The novel coronavirus (COVID-19) pandemic has drastically changed how patients are evaluated and treated and how they access ambulatory health care. Since there are currently no effective treatments or vaccines to prevent COVID-19, focus is placed on infection prevention through social distancing and quarantine. The Centers for Disease Control and Prevention (CDC) have set forth recommendations to prevent infections in healthcare settings by decreasing or eliminating non-urgent office visits. Telehealth refers to any healthcare process that occurs remotely, including provider training or team meetings, whereas telemedicine specifically describes using technology to connect a patient to a provider. To enable patients to retain access to healthcare, many countries have revised regulations to allow health care providers to use telemedicine and receive appropriate reimbursement [1]. For example, the Centers for Medicare and Medicaid Services in the USA have broadened access to, and reimbursement for, telemedicine services, allowing Female Pelvic Medicine and Reconstructive Surgery (FPMRS) providers the opportunity to provide continuity of care to existing patients who would otherwise remain disconnected.

In the field of FPMRS, telemedicine can limit community exposure to the most vulnerable population while simultaneously granting patients the opportunity to establish or continue care with a provider [2]. However, no clear guidelines exist regarding administering remote care for FPMRS patients.

Our objective was to conduct an expedited review of the evidence and to provide guidance for management of common outpatient urogynecologic conditions to help guide our specialty as we transition the way we provide care during the COVID-19 pandemic.

\section{Materials and methods}

Members of the Society of Gynecologic Surgeons (SGS) Collaborative Research in Pelvic Surgery consortium (SGS CoRPS) and the SGS Systematic Review Group (SGS SRG) participated in this project. The SGS CoRPS and SRG include members with expertise in clinical, surgical, and research management in FPMRS as well as systematic reviews and guideline development. No Institutional Review Board approval was required for this work.

We devised a list of questions and scenarios that FPMRS providers are likely to face as they engage patients virtually. We grouped these scenarios into diagnoses that would (1) likely require different treatment with telemedicine compared with in-person treatment or (2) would utilize accepted behavioral counseling and not deviate from current management paradigms. Expedited literature reviews were performed for four scenarios in which virtual management of patients would differ from direct visits [telemedicine in FPMRS patients, pessary management, urinary tract infection (UTIs), and urinary retention]. For scenarios in which the management via telemedicine would be similar to traditional conservative management (urinary and fecal incontinence, prolapse, defecatory dysfunction, and fecal incontinence), established algorithms and existing systematic reviews of conservative management were reviewed and summarized. Finally, expert consensus compiled and summarized the following; FPMRS conditions that are amenable to telemedicine management, urgent situations requiring in-person visits, symptoms that should alert FPMRS providers for possible COVID-19, and what FPMRS providers should consider when caring for patients with suspected or diagnosed COVID-19.

The methods, criteria, and literature flow for the expedited literature reviews, and salient meta-analysis details are reported in Appendix 1 [3]. Bullet-pointed summaries of our expedited literature reviews and expert consensus are listed in the body of this article. Additional information and details regarding the literature supporting these summaries can be found in Appendix 2.

\section{Results}

\section{Telemedicine in FPMRS patients}

The adoption and integration of telemedicine into a urogynecology practice is now possible, thanks to rapid advances in communications technology and widespread wireless access in many modern households. Still, FPMRS patient populations are diverse in age, socioeconomic status, and health literacy, and technologic devices and internet access are not universally available. Therefore, a multidimensional approach is necessary to provide a variety of options for patients seeking urogynecologic care.

Based on review of the literature (9 studies) [4-12] and expert consensus (EC):

\section{Patient satisfaction}

- Virtual visits provide similar patient satisfaction by building strong therapeutic relationships with patients through 
education, active listening, and shared decision-making [9].

- FPMRS patients living in rural settings may be more likely to attend follow-up visits when conducted remotely, although providers must consider limited internet access and technical capabilities for some elderly patients [12].

\section{Postoperative care}

- Patients whose postoperative visits are conducted using telemedicine reported high levels of satisfaction and experienced no increase in adverse events, emergency room visits, or primary care visits [10].

- Postoperative patients after midurethral slings with no symptoms of incontinence or after native tissue pelvic organ prolapse repairs can be appropriately assessed with telephone follow-up $[4,10]$.

\section{General principles for FPMRS telemedicine}

- Established patients not requiring a physical examination are ideal candidates for virtual visits (EC).

- New patients appreciate establishing a relationship with a provider, even before an in-person visit is possible, and will benefit significantly from non-surgical treatment options [7].

- Patients whose surgery has been canceled because of COVID-19 can replace their scheduled preoperative visit with a virtual discussion of alternative therapies as well as provide an opportunity for public health education related to COVID-19. In addition, rescheduling the patient's surgery will confirm a plan for providing definitive care. Alternatively, previously scheduled preoperative visits could be held as patients are likely to eventually have surgery (EC).

- There are many existing society websites with handouts and videos that can be used to supplement patient counseling (EC). They are available in many languages and in large print format. Some examples are:

- International Urogynecological Association (IUGA) Patient Leaflets [13], https://www.yourpelvicfloor. org/leaflets/

- American Urogynecologic Society, (AUGS) Voices for Pelvic Floor Disorders [14], https://www. voicesforpfd.org/resources/fact-sheets-anddownloads/

- National Association for Continence [15], https:// www.nafc.org/learning-library

\section{Regulatory access to telemedicine Services in the US}

Until COVID-19, telemedicine had not been utilized in most clinical settings. To expedite its use in the US, the Stafford Act, enacted in mid-March 2020, enabled the Centers for Medicare and Medicaid Services (CMS) to broaden access for Medicare telemedicine services. See Table 1 for CMS guidance to billing.

\section{Pessary management}

Seven studies provided data on risk of adverse events with long-term pessary use (without removal or cleaning) [17-23]. Nine additional articles were reviewed that provided information of interest during the pandemic [24-32]. Our analysis included three randomized controlled trials, three prospective cohorts, and one retrospective cohort. By metaanalysis (see Appendix 1), we estimated the following risks with continuous pessary use (no interval cleaning or examination) between 6 and 24 months: vaginal erosion or bleeding $5.0 \%$ (95\% CI 1.9, 9.0), vaginal discharge 5.8\% (95\% CI 3.6, 8.5 ), vaginitis $1.8 \%$ (95\% CI $0.2,4.6$ ), voiding dysfunction $4.7 \%$ (95\% CI 1.4, 9.8), and fistula $0 \%$ (95\% CI 0, 1.1).

Based on review of the literature (16 studies) and expert consensus:

Table 1 CMS guidance for billing during the COVID-19 pandemic*

\begin{tabular}{|c|c|c|c|}
\hline $\begin{array}{l}\text { Type of } \\
\text { service }\end{array}$ & What is the service? & $\begin{array}{l}\mathrm{HCPCS} / \mathrm{CPT} \\
\mathrm{CODE}\end{array}$ & $\begin{array}{l}\text { Type of } \\
\text { visit }\end{array}$ \\
\hline Video visit & $\begin{array}{l}\text { A video visit between a } \\
\text { provider and a patient } \\
\text { (billed by time) }\end{array}$ & $\begin{array}{l}\text { 99,201-99,215 } \\
\text { (Office or } \\
\text { other } \\
\text { outpatient } \\
\text { visits) }\end{array}$ & $\begin{array}{l}\text { For new or } \\
\text { establis- } \\
\text { hed } \\
\text { patients }\end{array}$ \\
\hline $\begin{array}{l}\text { Telephone or } \\
\text { electronic } \\
\text { visit } \\
\text { (e-visit) }\end{array}$ & $\begin{array}{l}\text { A phone or secure portal } \\
\text { communication } \\
\text { between a patient and } \\
\text { provider }\end{array}$ & $\begin{array}{l}99,421 \\
(5-10 \mathrm{~min}) \\
99,422 \\
(11-20 \mathrm{mi}- \\
\mathrm{n}) \\
99,423(\geq 21 \\
\text { minu })\end{array}$ & $\begin{array}{l}\text { For new or } \\
\text { establis- } \\
\text { hed } \\
\text { patients }\end{array}$ \\
\hline
\end{tabular}

CMS $=$ Centers for Medicare and Medicaid Services

HCPCS $=$ health care common procedural coding system

$\mathrm{CPT}=$ current procedural terminology

*Starting March 6, 2020, and for the duration of COVID-19 Public Health Emergency, CMS will enforce payments for Medicare telemedicine services furnished to beneficiaries in any healthcare facility and in their home. In telemedicine visits, the following must be documented: patient consented, use of video vs. audio (and reason if video not used), if video then general appearance of the patient, if audio the impression of how the patient sounds, and minutes spent in the visit [16]. Use of a specific telemedicine encounter is preferred for tracking and reimbursement purposes over a telephone encounter (typically used for sharing of discrete information) 
- Patients can safely extend the time interval between pessary cleanings to 6 months (and, in some cases, up to 24 months) with minimal risk of adverse events [17-23].

- Patients capable of pessary removal and reinsertion should be encouraged to self-clean their pessary [27, 31-35].

- Providers should consider empiric vaginal estrogen to minimize adverse events for patients not already using vaginal estrogen [17, 18, 28, 31, 33].

- For patients reporting copious vaginal discharge or bleeding, it may be appropriate to encourage home self-removal and to observe for symptoms such as voiding dysfunction until patients can safely be evaluated in the office (EC).

- Empiric treatment for bacterial vaginosis could be considered (EC)

\section{Empiric treatment of UTI}

In total, 60 articles provided information. Twenty-three contributed to the narrative summary and are cited in the paper. These included 2 RCTs [36, 37], 13 nonrandomized comparative studies [6, 8, 38-48], and 7 single group studies [49-55], and the remaining articles [5, 7, 39, 41, 43, 46, 50, 53, 56-83] included consensus documents, cost-effectiveness analyses, and narrative reviews.

Of note, most of our literature review and review of expert opinion was in line with the International Guidelines from the Infectious Disease Society of America (IDSA) and European Society for Microbiology and Infectious Disease (ESMID) recommendations, including choice of antibiotic for first-line therapy [84]. With recurrent UTI patients, although recent recommendations by the American Urological Association (AUA), Canadian Urological Association (CUA), and Society of Urodynamics, Female Pelvic Medicine, and Urogenital Reconstruction (SUFU) cited grade C evidence for cultures with every episode, the same level of evidence supports offering patient-initiated treatment when awaiting urine cultures [65].

Based on review of the literature (60 articles) and expert consensus:

- Telemedicine with empiric antibiotic therapy is effective and lowers costs, but results in more prescribing and therefore may negatively impact antibiotic resistance $[5,8,6$, 57-60, 76].

- The symptoms of dysuria, worsening frequency or urgency, gross hematuria, and lack of vaginal symptoms are significantly predictive of the presence of a UTI [44, 61].

- Prior culture results within the past year correctly correspond to subsequent cultures and sensitivities and thus should be used to guide empiric therapy, even in neurogenic bladder patients $[47,48,52,62]$.
- Patient factors such as age (> 65 years), immunosuppression, diabetes mellitus, catheter use, UTIs in the last year, and recent exposure to antibiotics should be assessed during telemedicine visits as these factors predict resistance to first-line antibiotics $[45,63,51,54,64]$. Fever and diabetes are risk factors for more severe infections or bacteremia and might guide treatment decisions about triage in person [43]. Providers should bear in mind that fever and various atypical symptoms may also indicate COVID-19 infection.

- Empiric treatments with either trimethoprimsulfamethoxazole (TMP-SMZ) or nitrofurantoin are costeffective choices [42, 56, 85-88]. Uncomplicated UTIs should be treated with one of the following empiric antibiotic strategies as supported by cost guidance, guidelines, and antimicrobial susceptibility: (1) TMP-SMZ 160/ $800 \mathrm{mg}$ orally twice daily for 3 days where antibiotic resistance does not exceed $20 \%$, (2) nitrofurantoin monohydrate macrocrystals $100 \mathrm{mg}$ orally twice daily for 5 days in patients with normal kidney function $(\mathrm{CrCl}$ $>30 \mathrm{ml} / \mathrm{min}$ ), particularly if there are contraindications or high resistance to TMP-SMZ, (3) fosfomycin $3 \mathrm{~g}$ once, or (4) pivmecillinam $400 \mathrm{mg}$ twice daily for 5 days [36, 40, $46,50,62,64,65,38,55,81,89,53,66,77]$.

- Antibiotic durations of 3-7 days are advisable and have better efficacy than single-dose therapy (with the exception of fosfomycin, which is an efficacious single dose regimen) $[67,72,90]$.

- Fluoroquinolone therapy should be reserved for higherrisk patients, locales where antibiotic resistance to alternative agents (particularly TMP-SMZ) exceeds $20 \%$, or when poor kidney function is known in the patient [39, 41, 68, 78-81, 91].

- Complicated UTIs in the current pandemic merit empiric treatment with a broader spectrum systemic fluoroquinolone antibiotic course to decrease hospital admissions, with plans to proceed to admission for parenteral antibiotics if severe symptoms occur or lack of response to oral antibiotics (e.g., intolerance to oral intake, high fever, severe pain, disorientation) $[49,69,70,92]$.

- Elderly patients and patients with diabetes should be given broader spectrum antibiotics (e.g., cephalosporins or fluoroquinolone therapy) for longer durations (7 days vs. single dose vs. 3 days) [53, 66, 71-73, 82, 83].

- Relief of symptoms can be used as a surrogate for UTI resolution in this pandemic (EC).

- Other strategies to avoid antibiotics could include fluid hydration, cranberry supplements, or bladder soothants (e.g., phenazopyridine) (EC).

- Laboratory alternatives include over-the-counter urine dipstick products, urine PCR [74], or utilizing remote laboratory locations to minimize exposure in hospital settings (EC). 
Table 2 Potential reasons for urgent visits in the FPMRS clinic during a pandemic

Reason Explanation

Acute pelvic pain or pelvic floor myalgia

Acute interstitial cystitis flare

Refractory or relapsing UTI

Postoperative concerns or complications

Acute retention

Pessary complications

New onset of genitourinary or rectovaginal fistula

Acute genitourinary postpartum complaints

Refractory vaginitis or vulvar complaints

Urethral or vaginal mass (other than prolapse)

Mesh complication
Severe pain or worsening pain/flare/need for trigger point injections

Flare or need for bladder instillation

Failed empiric antibiotics or complicating symptoms such as fever or concern for pyelonephritis

Examples: persistent nausea/vomiting, concern for urinary retention, heavy vaginal bleeding, severe abdominal pain, concern for infection or cuff dehiscence

See voiding dysfunction and retention section

Pessary patient with persistent or significant vaginal bleeding or pain

Consider if evaluation in office may change your management (e.g., transurethral catheter or antibiotics)

Separation of 3rd/4th degree perineal laceration, acute retention, or concern for a fistula

Failed empiric treatment

In cases with acute retention or concern for mass causing obstruction, when examination can facilitate referral to a specialist (e.g., gynecologic oncologist)

New onset complaint or worsening symptoms
- Strategies to avoid UTIs that do not require in-person visits include vaginal estrogen or use of D-mannose $1000 \mathrm{mg}$ twice daily [75] (EC).

- Recurrent UTI patients may be offered patient-initiated treatment based on past urine cultures, as supported by grade $\mathrm{C}$ evidence in the AUA/CUA/SUFU guidelines. They further recommend culture with every episode but this may be suspended during the COVID-19 pandemic when the risk of healthcare exposure outweighs the need for culture [65] (EC).

\section{Voiding dysfunction and retention}

We found 23 articles, of which 10 had data extracted [93-102]. Thirteen additional articles provided information pertinent to management of voiding dysfunction during this pandemic [103-115].

Based on review of the literature (23 articles) and expert consensus:

- Chronic urinary retention (PVR $>300 \mathrm{ml}$ for $\geq 6$ months) puts patients at risk of upper urinary tract injury. Imaging

Table 3 Perioperative considerations: non-elective cases

\begin{tabular}{|c|c|}
\hline $\begin{array}{l}\text { Operative } \\
\text { state }\end{array}$ & Recommendation \\
\hline Preoperative & $\begin{array}{l}\text { - Screen all patients for symptoms upon arrival to the healthcare setting; triage for testing based on symptoms } \\
\text { - Consider universal testing once availability of COVID-19 kits increases } \\
\text { - Consider a chest x-ray as a screening tool since it may be obtained faster than confirmatory testing }\end{array}$ \\
\hline Intraoperative & $\begin{array}{l}\text { - For COVID-19-positive cases: } \\
\text { - Operating room staff should have full personal protective equipment, and only essential personnel should be present } \\
\text { - Limit movement of personnel in and out of the OR especially during airway management given the increased risk of aerosolization of } \\
\text { viral particles } \\
\text { - Consider laparotomy vs. laparoscopy by balancing the risks of surgical morbidity to the patient with risk of viral transmission to the } \\
\text { provider } \\
\text { - Limit use of electrocautery unless absolutely necessary for patient safety. If used, electrocautery devices should be used at the lowest } \\
\text { effective setting and in conjunction with deliberate smoke suctioning with filters when energy is used } \\
\text { - Insufflation during laparoscopic cases should be kept to a minimum and completely evacuated through a filtration system prior to trocar } \\
\text { venting or removal }\end{array}$ \\
\hline Postoperative & $\begin{array}{l}\text { - Same-day discharge should be encouraged to avoid prolonged hospitalization and reduce hospital burden } \\
\text { - For COVID-19-positive cases: } \\
\text { - Consider prophylaxis or treatment doses of anticoagulation as COVID-19 infections may produce a hypercoagulable state }\end{array}$ \\
\hline
\end{tabular}


and/or laboratory evaluation along with appropriate catheterization should be considered [111].

- Factors that suggest a patient is at low risk for postoperative urinary retention (following pelvic surgery) include: voiding $>200 \mathrm{ml}$ after being retrograde filled with $300 \mathrm{ml}$, voiding $\geq 50 \%$ of the retrograde-filled volume, and women who subjectively feel that the postoperative force of their urinary stream is at least $50 \%$ of their baseline force of stream [102, 108, 112].

- Regional anesthesia is unlikely to substantially increase the risk of postoperative urinary retention and can be considered for vaginal surgery in an effort to decrease the potential risk of aerosolization of COVID-19 with general anesthesia [113] (EC).

- Clean intermittent self-catheterization (CISC) may be preferable to an indwelling catheter for urinary retention when possible [104, 107, 108]. Risk factors that predict poor success with CISC include obesity, poor dexterity, cognitive impairment, and pain with catheterization [105-107].

- When patients call with symptoms of possible urinary retention, consider instruction in behavioral modification prior to recommending CISC. This includes encouraging the patient to create a relaxing environment with adequate time for voiding while taking slow deep breaths and relaxing their pelvic floor muscles [94]. Patients could also be instructed to double or triple void [116] or in the use of the Crede maneuver (expert consensus).

- If behavioral modifications fail, patients should be given the option of CISC. A prescription for catheters can be called into a pharmacy (delivery may be available), and remote teaching of the CISC technique can be attempted. If video conferencing is available, patients could be taught to use a clean technique with a mirror. Initially, the patient lies down and inserts a small-gauge (e.g., 10, 12, or 14 French) catheter. When proficient with the mirror, she can be instructed to insert the catheter by feel in the sitting or standing position. Online instructional videos are also available (https://vimeo. com/261183016) $[95,117]$ and online patient handouts are available as well (https:/www.yourpelvicfloor.org/media/ Intermittent_Self_Catheterization.pdf) [13] (https://www. voicesforpfd.org/assets/2/6/ISC.pdf) [13, 14] (EC).

- Patients with postoperative urinary retention who need indwelling catheterization can be instructed regarding safe removal of the catheter on postoperative day 7 at home without an office visit by cutting the balloon port and/or desufflating the catheter balloon. Consider having the patient remove the catheter early in the day to allow for an inperson office visit later on the same day if necessary [114].

- While antibiotics may reduce the incidence of asymptomatic bacteriuria during short-term catheter use, there is no strong evidence supporting the use of prophylactic antibiotics after hospital discharge in women being catheterized for postoperative urinary retention $[96,97]$.
- Antibiotic prophylaxis should not be routinely used in patients with long-term catheterization, and there is insufficient evidence to make recommendations about routine catheter change (e.g., every 2-4 weeks) in patients with long-term indwelling transurethral catheterization [115].

- There is no strong evidence supporting the use of oral medication (e.g., alpha-adrenergic antagonists) in the treatment of voiding dysfunction or urinary retention in women $[93,99,100]$.

\section{Urinary incontinence}

A recent systematic review was published on treatment options for women with urinary incontinence [118]. This systematic review focused on studies of adult women with stress urinary incontinence (SUI), urgency urinary incontinence (UUI), or mixed urinary incontinence (MUI); women were excluded if they were pregnant or hospitalized. We updated this review with additional studies published since August 2018 [118-124].

Based on this recent systematic review, six additional studies [118-124], and expert consensus:

- SUI, UUI, and MUI can be discussed and treated with telemedicine (EC).

- Behavioral therapy including bladder training, pelvic floor physical therapy or Kegel exercises, weight loss, and yoga have demonstrated significant improvement and/or complete resolution of SUI and UUI symptoms [125].

- Patients can implement behavioral interventions without leaving home (EC).

- Patient-initiated options such as incontinence tampons (Poise Impressa ${ }^{\circledR}$ ) or a patient fitted pessary (Uresta $®$ ) could be recommended $[119,124]$.

- Patients currently treated with third-line treatments for UUI such as intradetrusor onabotulinum toxin A or percutaneous tibial nerve stimulation could revert back to behavioral modifications and medications (anticholinergic or $\beta_{3}$-adrenoceptor agonist) until they can return for inperson office visits (EC).

- Consider balancing the risk of exposure to COVID-19 versus the risk of dementia from anticholinergic use [120]. It is unlikely that short-term use during healthcare interruption due to this pandemic will result in long-term dementia effects (expert consensus).

- Consider the risk of hypertension with $\beta_{3}$ adrenoceptor agonists. However, two recent systematic reviews reported no difference in hypertension risk between mirabegron and placebo [121, 122]. 
- Smartphone applications (apps) can be used to help teach and track Kegel exercises [123].

- $\quad$ The free app Kegel Trainer or paid app Kegel Trainer Pro® were the highest rated apps based on a recent review [123].

\section{Pelvic organ prolapse, defecatory dysfunction, and fecal incontinence}

Existing AUGS Best Practice guidelines, American College of Obstetricians and Gynecologists (ACOG) guidelines, American Society of Colon and Rectal Surgeons (ASCRS) clinical practice guidelines, and systematic reviews were summarized to guide treatment of prolapse, fecal incontinence, and defecatory dysfunction via telemedicine [126-129]. Pelvic organ prolapse can be challenging to evaluate without a physical examination. However, the virtual visit provides an opportunity to counsel patients on the pathophysiology, possible treatment options, and techniques to prevent progression. Similarly, for defecatory dysfunction and fecal incontinence, conservative measures listed in the table can be initiated to help alleviate patients' symptoms. It is important to note that a change in bowel habits, weight loss, and rectal bleeding may warrant referral to a gastroenterologist or colorectal surgeon to rule out colorectal cancer [128, 129]. Of note, if a patient reports new onset fecal incontinence or acute worsening of fecal incontinence, she should be screened for other COVID-19 symptoms and then referred for the appropriate care, as diarrhea is a possible symptom of COVID-19.

\section{Pelvic organ prolapse}

- Only 10-20\% of women will have an increase in prolapse stage over 2 years; therefore, most patients can be reassured regarding delay in surgical or pessary management $[130,131,129]$.

- Weight loss, reducing activities that strain the pelvic floor, smoking cessation, and avoiding constipation may improve symptoms and decrease progression of prolapse [132].

- Pelvic Floor muscle training and exercises can decrease prolapse in some patients [132, 133].

- For pelvic muscle exercises, providers may suggest online instructions (https://www.yourpelvicfloor.org/ media/Pelvic Floor Exercises RV2-1.pdf) [13] (https://www.voicesforpfd.org/assets/2/6/Bladder Training.pdf) [14]. Home biofeedback devices can be used, such as Leva®, which is an FDA-cleared pelvic floor muscle trainer with visualization technology, smartphone applications, vaginal weights, virtual pelvic floor therapy appointments, or internet pelvic floor training (EC).

- Encouraging patients to splint or insert a large tampon may help alleviate symptoms in cases of prolapse causing incomplete bladder emptying (EC).

\section{Defecatory dysfunction}

- Dietary changes and fiber supplementation (insoluble fiber) can improve stool consistency and help with stool evacuation [126, 127].

- Osmotic or stimulant laxatives can help defecatory dysfunction and postoperative constipation [126].

- Position changes during bowel movements or a squatty potty can improve defecation [134].

- Splinting vaginally or at the perineum may help women with incomplete evacuation from a rectocele (EC).

\section{Fecal incontinence}

- Protective devices can be utilized [127]. These include pads or adult diapers, adhesive patches (e.g., butterfly pads), and skin care with protective ointments that are zinc based (EC).

- A food diary can be used to identify triggers to avoid [127]. Triggers associated with loose stool can include sugar replacements, caffeine, and lactose.

- Medications that may cause loose stool should be avoided [135]. Some common medications that cause diarrhea include: antacids, proton pump inhibitors, antibiotics, SSRIs, beta blockers, ACE inhibitors, metformin, and cholestyramine.

- Dietary fiber (soluble) with increased fluid intake can provide more bulk to the stool and help achieve the ideal stool consistency [126, 136].

- Consider medications $([126,127])$ to treat loose stools and help FI: [126, 127].

- Bowel schedules, tap water enemas, glycerine, or bisacodyl suppositories can help patients to reliably evacuate the rectum [126].

- A systematic review found anal plugs (not offered in the US) are poorly tolerated but effective [137].

\section{Urgent situations}

The COVID-19 pandemic presents a challenge for both patients and providers to determine the appropriate scenario requiring a more thorough evaluation, examination, and/or laboratory testing. When a provider is considering the necessity of an in-office visit, they must weigh the 
risks of COVID-19 exposure taking into account the current status of the outbreak in that specific region, the severity of the patient complaint, as well as the age and comorbidities of the patient. It appears that older age, diabetes, and immunosuppression increase the risk of morbidity and mortality associated with COVID-19 infection [138]. As there are no guidelines on clinic visits during a pandemic for this specialty, group consensus was obtained, and a list of reasons that may require an inperson visit was generated (Table 2). Providers should also consider a clinic visit if there is a reasonable chance a physical examination or office diagnostic testing may change the course of treatment for an urgent complaint. One must also consider that the course of the COVID-19 pandemic will change over time, which might impact these recommendations.

\section{COVID-19-specific concerns for FPMRS patients}

Patients seen by urogynecologists are likely to have risk factors that increase the chance of complications from COVID-19. Thus, it is important for FPMRS providers to be aware of COVID-19 symptoms that should prompt a referral for further evaluation and testing. For example, upper respiratory symptoms and bowel changes are possible presenting symptoms for COVID-19. A patient with an increase in their stress incontinence due to a dry cough or worsened fecal incontinence due to diarrhea should trigger consideration for further COVID-19 screening based on the regional protocol.

The American College of Surgeons (ACS) has advised postponing elective cases until after the acute COVID-19 crisis abates [139]. General guidance to assist FPMRS and other surgical specialties with staged postponement of surgical cases has been published [140]. During the pandemic, there will be a need for urgent surgical intervention in some situations, and a plan for management of these non-elective cases is required. A brief review of perioperative considerations for non-elective cases including COVID-19 positive cases was generated (Table 3) [141, 142]. When discussing surgical intervention with patients negative for COVID-19 infection, surgeons should discuss the unique risks of nosocomial COVID-19 infection during the consent process, including the efforts undertaken to protect the patient and the challenges of preventing contamination. Also consideration should be placed on ERAS and same-day discharge to decrease risk and exposure to patients.

\section{Discussion}

In this review, we have explored conditions that FPMRS providers are likely to face as they engage patients virtually during the COVID-19 pandemic. We have reviewed the literature and summarized our findings in the sections above. Overall, behavioral and conservative management will be valuable as first-line treatments provided in a virtual setting (via phone or internet communication). There are situations that will require different treatments in the virtual setting than in person, and there are some that will require an in-person visit despite the risks of COVID-19 exposure and spread.

The strengths of this review include our use of expedited evidence review methods as well as the author team's experience conducting systematic reviews and developing clinical practice guidelines, along with its advanced expertise in FPMRS. The main limitations to this review are the rapid nature of the review and the lack of data regarding many of the pertinent clinical questions. Our expedited evidence methods inevitably missed salient studies. Furthermore, the COVID-19 pandemic is changing our world day by day, and it is impossible to forecast how this will impact our management of common FPMRS conditions in the months to come.

The COVID-19 pandemic is unprecedented in terms of the scope and impact on the world's healthcare systems. To control and prevent the spread of infection, FPMRS practices will need to utilize telemedicine to safely provide continuity of care to our patients. We have provided literature and expertbased guidance for the practicing FPMRS.

Acknowledgements This work was conducted by the Society of Gynecologic Surgeons Collaborative Research in Pelvic Surgery Consortium (SGS CoRPS) and Systematic Review Group (SRG).

Support Funding provided by the Society of Gynecologic Surgeons (SGS) supports assistance by methods experts in systematic reviews and other logistics.

Author contributions Grimes: protocol, project development, data collection or management, data analysis, manuscript writing/editing.

Balk: protocol, project development, data collection or management, data analysis, manuscript writing/editing.

Crisp: protocol, project development, data collection or management, data analysis, manuscript writing/editing.

Antosh: protocol, project development, data collection or management, data analysis, manuscript writing/editing.

Murphy: protocol, project development, data collection or management, data analysis, manuscript writing/editing.

Halder: data collection or management, data analysis,manuscript writing/editing.

Jeppson: data collection or management, data analysis, manuscript writing/editing.

Weber LeBrun: data collection or management, data analysis, manuscript writing/editing. 
Raman: data collection or management, data analysis, manuscript writing/editing.

Kim-Fine: data collection or management, data analysis, manuscript writing/editing.

Iglesia: data collection or management, data analysis, manuscript writing/editing.

Dieter: data collection or management, data analysis, manuscript writing/editing.

Yurteri-Kaplan: data collection or management, data analysis, manuscript writing/editing.

Adam: project development, data collection or management, data analysis.

Meriwether: protocol, project development, data collection or management, data analysis, manuscript writing/editing.

\section{Compliance with ethical standards}

Financial disclaimers/conflict of interest Grimes: Expert testimony for Johnson and Johnson.

Meriwether: Consultant for RBI medical, Travel reimbursements from SGS (voting board member as Research Chair), Book editing/authorship royalties from Elsevier.

Jeppson: Consultant for Johnson \& Johnson.

Balk: Consultant for society for Gynecologic Surgeons and American Association of Gynecologic Laparoscopists.

Murphy: Expert witness for Johnson and Johnson, Boston Scientific, President of Soceity of Gynecologic Surgeons.

No conflicts: Balk, Crisp, Antosh, Adam, Weber LeBrun, Halder, Kim-Fine, Dieter, Raman, Yurteri-Kaplan, Iglesia.

\section{Appendix 1}

\section{Literature review methods}

We conducted expedited literature reviews on four topics: (1) telemedicine, (2) pessary use, (3) empiric therapy for urinary tract infections (UTIs), and (4) dysfunctional urinary voiding (urinary retention).

For the expedited literature reviews we modified standard systematic review methods used by the SGS SRG and the Agency for Healthcare Research and Quality Evidence-based Practice Center Program [3]. Briefly, we first determined which among all the covered topics were amenable for literature review (and had not been recently addressed by existing systematic reviews or guidelines). For each of these four topics, we developed eligibility criteria by consensus. Based on these criteria, four formal literature searches were developed and run in PubMed, the Cochrane Central Register of Controlled Trials, and the Cochrane Database of Systematic Reviews. Literature searches were conducted from database inception through March 29, 30, or 31, 2020. All searches were restricted to English language publications and excluded case reports, animal studies, and non-research articles (except narrative reviews). For all topics, we sought existing systematic reviews, primary studies, and pertinent narrative reviews.

Each literature search was entered into Abstrackr software (http://abstrackr.cebm.brown.edu/) and single screened by members of the SRG and CoRPS. Accepted abstracts were rescreened in Abstrackr by topic leaders. Remaining potentially relevant citations were entered into Google Sheets spreadsheets available to all researchers for tracking and basic data extraction. Immediately available full-text articles were retrieved and rescreened for eligibility by team members.

Data from studies of long-term pessary use adverse events were extracted into a Google Sheet file to capture study and pessary characteristics and event rate data. For other topics, team members culled pertinent information from relevant articles.

\section{Telemedicine in FPMRS patients}

Regarding telemedicine, we sought articles on effective approaches, and pitfalls, of telemedicine, virtual healthcare, and care by telephone for women with urogynecologic issues (e.g., urinary or defecatory incontinence, urinary or defecatory voiding dysfunction, pelvic organ prolapse, and UTI).

\section{Research question}

1. Are any virtual visit platforms tested with FPMRS patients or older women?

\section{Study eligibility criteria (PICOS)}

\section{Population}

- Urogynecology

- Female Pelvic Medicine and Reconstructive surgery (FPMRS) care

- Incontinence, urinary

- Incontinence, defecatory

- Prolapse

- Recurrent UTIs

- Defecatory dysfunction, including obstructed defecation

- Urinary voiding dysfunction, including retention

\section{Interventions}

- Virtual healthcare, including telemedicine/telehealth, videoconference, telephone, Web-based, app-based, rural healthcare (regarding long-distance care) 


\section{Outcomes}

- (Primary) emergency/urgent in-person care

- (Primary) adverse outcomes/complications

- (Secondary) clinical (patient-centered) outcomes

- (Secondary) receiving urogynecology care

\section{Study design/article availability}

- Primary studies of any design except case reports and case series

- Systematic review or guideline

- $\quad N \geq 10$ per intervention (group)

- English language publication

- Article immediately available for review

Literature search strategies Inception through March 30, 2020.

MEDLINE via PubMed.

("Telecommunications"[Mesh] or teleconsult* or Telemedicine or "Mobile Health" or mHealth* or Telehealth* or telerehabilitat* or eHealth or e-health or "rural health" or "Rural Health Services"[Mesh] OR "Telemedicine"[Mesh] OR (("Patient Care"[Mesh] OR "Patient Care" OR "Therapeutics"[Mesh] OR therapy OR therapeutic OR "Health Services"[Mesh] OR health OR “Diagnosis" [Mesh] OR diagnosis OR "Professional-Patient Relations"[Mesh] OR "Patient Relations" OR "Health Services Accessibility"[Mesh] OR "Health B ehavior"[Mesh]) A N D ("Telecommunications"[Mesh] OR "Computer Communication Networks"[Mesh] or e-medicine or email or e-mail or Videoconferenc* or wireless or phone* or telephone*)))

\section{AND}

("Uterine prolapse" OR "Vaginal prolapse" OR "Pelvic Organ Prolapse" OR "Urogenital Prolapse" OR "Vaginal Vault Prolapse" OR "Cystocele" OR "cystocoele" OR "Rectal Prolapse" OR "Rectocele" OR "rectocoele" OR "Visceral Prolapse" OR "Uterine Disease" OR “Overactive Bladder" OR "Overactive Detrusor Function" OR "Urinary incontinence" OR "detrusor instability" OR "Urinary Tract Infection" OR "Pyuria" OR "Urinary Retention" OR "Fecal incontinence" OR "Bowel incontinence" OR "Fecal soiling" OR “obstructed defecation" OR "Defecatory dysfunction" OR "Female Pelvic Medicine and Reconstructive surgeon" OR "urogynecology" OR "Uterine Prolapse"[Mesh] OR "Pelvic Organ Prolapse"[Mesh] OR “Uterine Diseases”[Mesh] OR “Urinary Bladder,
Overactive"[Mesh] OR "Urinary Incontinence"[Mesh] OR "Urinary Tract Infections"[Mesh] OR "Fecal Incontinence"[Mesh] OR "Constipation"[Mesh] OR "Urinary Retention"[Mesh])

NOT

("address"[pt] or "autobiography"[pt] or "bibliography”[pt] or "biography"[pt] or "case reports" $[\mathrm{pt}]$ or "comment" $[\mathrm{pt}]$ or "congress" $[\mathrm{pt}]$ or "dictionary" $[\mathrm{pt}]$ or "directory" $[\mathrm{pt}]$ or "festschrift" $[\mathrm{pt}]$ or "government publication" $[\mathrm{pt}]$ or "historical article" $[\mathrm{pt}]$ or "interview” $[\mathrm{pt}]$ or "lecture" [pt] or "legal case" [pt] or "legislation" $[\mathrm{pt}]$ or "news" $[\mathrm{pt}]$ or "newspaper article"[pt] or "patient education handout" [pt] or "periodical index" [pt] or "comment on" or "case report" [pt] or "case series"[pt] or ("Animals"[Mesh] NOT "Humans"[Mesh]) OR rats[tw] or rat[tw] or cow [tw] or cows[tw] or chicken*[tw] or horse[tw] or horses[tw] or mice[tw] or mouse[tw] or bovine[tw] or sheep[tw] or ovine [tw] or murinae[tw] or cats[tw] or cat $[\mathrm{tw}]$ or $\operatorname{dog}[\mathrm{tw}]$ or $\operatorname{dogs}[\mathrm{tw}]$ or rodent $[\mathrm{tw}]$ )

Limit to English.

\section{Cochrane databases.}

(teleconsult or Telemedicine or Mobile Health or mHealth or Telehealth or telerehabilitation or eHealth or e-health or "rural health" OR Telecommunication OR Computer or e-medicine or email or e-mail or Videoconference or wireless or phone or telephone)

AND

(Uterine prolapse OR Vaginal prolapse OR Pelvic Organ Prolapse OR Urogenital Prolapse OR Vaginal Vault Prolapse OR Cystocele OR cystocoele OR Rectal Prolapse OR Rectocele OR rectocoele OR Visceral Prolapse OR Uterine Disease OR Overactive Bladder OR Overactive Detrusor Function OR Urinary incontinence OR detrusor instability OR Urinary Tract Infection OR Pyuria OR Urinary Retention OR Fecal incontinence OR Bowel incontinence OR Fecal soiling OR obstructed defecation OR Defecatory dysfunction OR Female Pelvic Medicine OR urogynecology)

\section{Literature and screening results}

The combined (partially deduplicated) searches yielded 3670 citations. These were screened singly in full by seven team members. Among these, 140 citations were screened in, which were rescreened by a single team member, who selected 15 for further review. Two citations referred to the same study, and three other articles were not available. In total, 11 full-text articles were reviewed, 9 of which were considered useful and are cited in the paper. 


\section{Pessary management}

Regarding pessary management, we sought studies that reported rates of adverse outcomes (erosion, vaginal bleeding, discharge, vaginitis, fistulas) in women with pessaries in situ for $>3$ months.

\section{Research questions}

1. How long can a pessary remain in place without removal/ cleaning?

2. What is the risk of complications (erosion, vaginal bleeding, discharge, vaginitis, fistulas) due to delayed removal, cleaning, and inspection of the vagina?

3. Is there benefit or reduction in adverse events by placing patients on vaginal estrogen if they are not already using it?

\section{Study eligibility criteria (PICOS)}

Note that the literature search focused on Pessary Question 2. Population

- Women with pessaries in place

\section{Intervention}

- Pessary in place for $\geq 4$ weeks

\section{Outcomes}

- Erosion

- Abrasion

- Vaginitis

- Vaginal infection

- Fistula

- Vaginal discharge

- Abnormal vaginal bleeding

- Urinary tract infection

- Other clinical adverse outcomes

\section{Study design/article availability}

- Primary studies of any design except case reports and case series

- Systematic review or guideline

- $\quad N \geq 10$ per intervention (group)

- English language publication

- Article immediately available for review
Literature search strategies Inception through March 29, 2020.

MEDLINE via PubMed.

((“Pessaries"[Mesh] OR pessary OR pessaries OR (gellhorn not gellhorn[author]) or "incontinence dish"))

NOT

("address"[pt] or "autobiography"[pt] or "bibliography"[pt] or "biography"[pt] or "case reports"[pt] or "comment" [pt] or "congress" [pt] or "dictionary" [pt] or "directory" [pt] or "festschrift" [pt] or "government publication"[pt] or "historical article"[pt] or "interview"[pt] or "lecture"[pt] or "legal case"[pt] or "legislation"[pt] or "news" [pt] or "newspaper article"[pt] or "patient education handout"[pt] or "periodical index"[pt] or "comment on" or "case report"[pt] or "case series"[pt] or ("Animals"[Mesh] NOT "Humans"[Mesh]) OR rats[tw] or rat[tw] or cow[tw] or cows[tw] or chicken*[tw] or horse[tw] or horses[tw] or mice[tw] or mouse[tw] or bovine[tw] or sheep[tw] or ovine [tw] or murinae $[\mathrm{tw}]$ or cats $[\mathrm{tw}]$ or cat $[\mathrm{tw}]$ or $\operatorname{dog}[\mathrm{tw}]$ or $\operatorname{dogs}[\mathrm{tw}]$ or rodent[tw])

Limit to English.

Cochrane databases.

[Pessaries] explode all trees OR pessary OR pessaries OR (gellhorn NOT (gellhorn):au) OR "incontinence dish."

\section{Literature and screening results}

The combined (partially deduplicated) searches yielded 1659 citations. These were screened singly in full by six team members. Among these, 140 citations were screened in, which were rescreened by a single team member, who selected 85 for further review of which 15 articles were not available in full text. Upon full-text review, seven studies reported data on adverse events related to long-term use of pessaries (without removal and cleaning); nine articles provided additional information. They are cited in the paper.

\section{Meta-analysis methods}

We conducted random-effects model restricted maximum likelihood meta-analyses of the proportions of women with adverse events. To account for non-normal distribution, proportions were double arcsine transformed. Meta-analyses were conducted in OpenMetaAnalyst (http://www.cebm. brown.edu/openmeta).

\section{Meta-analysis results}

Seven studies (with 9 study arms) were included in meta-analyses. These are summarized in Appendix Table 4 
Table 4 Studies reporting adverse outcomes with long-term pessary use (without removal and cleaning)

\begin{tabular}{|c|c|c|c|c|c|c|c|}
\hline Outcome & Study & $\begin{array}{l}\text { Pubmed } \\
\text { ID }\end{array}$ & Country & $\begin{array}{l}\text { Pessary } \\
\text { type }\end{array}$ & $\begin{array}{l}\text { Duration } \\
\text { (months) }\end{array}$ & Outcome definition & $\mathrm{n} / \mathrm{N}(\%)$ \\
\hline \multirow[t]{3}{*}{ Discharge } & $\begin{array}{r}\text { Cheung } \\
2016\end{array}$ & $27,275,798$ & China & Ring & 6 & Unusual or bothersome discharge & $6 / 132(4.5 \%)$ \\
\hline & Miceli 2020 & $32,062,679$ & Spain & Ring & 24 & Increase in vaginal discharge & $6 / 115(5.2 \%)$ \\
\hline & Chien 2019 & $31,393,340$ & Taiwan & Gellhorn & $\begin{array}{l}12-111 \\
(\text { mean } 50.4)\end{array}$ & Abnormal vaginal discharge & $8 / 93(8.6 \%)$ \\
\hline \multirow[t]{9}{*}{ Erosion } & Propst 2020 & $31,809,432$ & USA & Multiple & 3 & Type 3 or 4 (epithelial break or erosion) & $4 / 54(7.4 \%)$ \\
\hline & Thys 2020 & $31,907,565$ & USA & Ring & 3 & Lesion of the vaginal epithelium & $0 / 132(0 \%)$ \\
\hline & Tam 2019 & $31,561,819$ & $\begin{array}{l}\text { Hong } \\
\text { Kong }\end{array}$ & Ring & 3 & Ulceration or erosion & $1 / 29(3.4 \%)$ \\
\hline & Propst 2020 & $31,809,432$ & USA & Multiple & 6 & Type 3 or 4 (epithelial break or erosion) & $1 / 57(1.8 \%)$ \\
\hline & Lone 2011 & $21,575,953$ & UK & Multiple & 6 & $\begin{array}{l}\text { Discontinue use because of excoriation or } \\
\text { bleeding }\end{array}$ & $5 / 223(2.2 \%)$ \\
\hline & Tam 2019 & $31,561,819$ & $\begin{array}{l}\text { Hong } \\
\text { Kong }\end{array}$ & Ring & 6 & Ulceration or erosion & $6 / 30(20 \%)$ \\
\hline & $\begin{array}{r}\text { Cheung } \\
2016\end{array}$ & $27,275,798$ & China & Ring & 6 & Abnormal vaginal bleeding & $9 / 132(6.8 \%)$ \\
\hline & Miceli 2020 & $32,062,679$ & Spain & Ring & 24 & Vaginal bleeding/erosion & $8 / 115(7.0 \%)$ \\
\hline & Chien 2019 & $31,393,340$ & Taiwan & Gellhorn & $\begin{array}{l}12-111 \\
(\text { mean } 50.4)\end{array}$ & Vaginal bleeding/erosion & $9 / 93(9.71 \%)$ \\
\hline \multirow[t]{8}{*}{ Fistula } & Propst 2020 & $31,809,432$ & USA & Multiple & 3 & Fistula & $0 / 54(0 \%)$ \\
\hline & Tam 2019 & $31,561,819$ & $\begin{array}{l}\text { Hong } \\
\text { Kong }\end{array}$ & Ring & 3 & Fistula & $0 / 29(0 \%)$ \\
\hline & Thys 2020 & $31,907,565$ & USA & Ring & 3 & Fistula & $0 / 132(0 \%)$ \\
\hline & Propst 2020 & $31,809,432$ & USA & Multiple & 6 & Fistula & $0 / 57(0 \%)$ \\
\hline & Tam 2019 & $31,561,819$ & $\begin{array}{l}\text { Hong } \\
\text { Kong }\end{array}$ & Ring & 6 & Fistula & $0 / 30(0 \%)$ \\
\hline & Lone 2011 & $21,575,953$ & UK & Multiple & 6 & Fistula & $0 / 223(0 \%)$ \\
\hline & Miceli 2020 & $32,062,679$ & Spain & Ring & 24 & Major complication & $0 / 115(0 \%)$ \\
\hline & Chien 2019 & $31,393,340$ & Taiwan & Gellhorn & $\begin{array}{l}12-111 \\
(\text { mean 50.4) }\end{array}$ & Fistula & $0 / 93(0 \%)$ \\
\hline \multirow[t]{4}{*}{ Retention } & Tam 2019 & $31,561,819$ & $\begin{array}{l}\text { Hong } \\
\text { Kong }\end{array}$ & Ring & 3 & Urinary retention & $0 / 29(0 \%)$ \\
\hline & Tam 2019 & $31,561,819$ & $\begin{array}{l}\text { Hong } \\
\text { Kong }\end{array}$ & Ring & 6 & Urinary retention & $0 / 30(0 \%)$ \\
\hline & $\begin{array}{r}\text { Cheung } \\
2016\end{array}$ & $27,275,798$ & China & Ring & 6 & Voiding difficulty, de novo & $\begin{array}{l}10 / 92 \\
\quad(10.9 \%)\end{array}$ \\
\hline & Chien 2019 & $31,393,340$ & Taiwan & Gellhorn & $\begin{array}{l}12-111 \\
(\text { mean } 50.4)\end{array}$ & Voiding/defecating difficulty & $4 / 93(4.3 \%)$ \\
\hline \multirow[t]{4}{*}{ Vaginitis } & Tam 2019 & $31,561,819$ & $\begin{array}{l}\text { Hong } \\
\text { Kong }\end{array}$ & Ring & 3 & Vaginitis & $0 / 29(0 \%)$ \\
\hline & $\begin{array}{r}\text { Cheung } \\
2016\end{array}$ & $27,275,798$ & China & Ring & 6 & Bacterial vaginosis & $1 / 132(0.8 \%)$ \\
\hline & Tam 2019 & $31,561,819$ & $\begin{array}{l}\text { Hong } \\
\text { Kong }\end{array}$ & Ring & 6 & Vaginitis & $3 / 30(10 \%)$ \\
\hline & Miceli 2020 & $32,062,679$ & Spain & Ring & 24 & Candida glabrata & $1 / 115(0.9 \%)$ \\
\hline
\end{tabular}


Studies

Cheung 2016 discharge

Miceli 2020 discharge

Chien 2019 discharge

Overall $\left(I^{2}=0 \%, P=0.45\right)$
$\mathrm{Ev} / \mathrm{Tr} t$

$0.0455(0.0167,0.0874)$

$0.0522(0.0192,0.1001)$

$0.0860(0.0379,0.1511)$

$6 / 132$

$6 / 115$

$8 / 93$

$0.0578(0.0355,0.0850) 20 / 340$

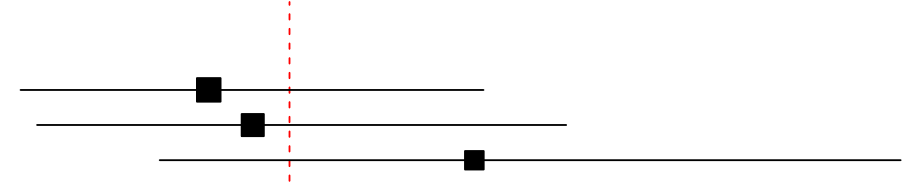

Fig. 1 Vaginal discharge meta-analysis

\begin{tabular}{lllcl}
\hline 0.02 & & & & \\
& 0.05 & & & \\
& Proportion & 0.08 & 0.12 & 0.15
\end{tabular}

\section{Studies}

Tam 2019 vaginitis 3 mo Cheung 2016 vaginitis Tam 2019 vaginitis 6 mo

Miceli 2020 vaginitis

Overall $\left(I^{2}=47.4 \%, P=0.12\right)$

Fig. 2 Vaginitis meta-analysis

$\begin{array}{ccc}\text { Estimate }(95 \% \mathrm{CI}) & \text { Ev/Trt } \\ 0.0167(0.0000,0.0921) & 0 / 29 \\ 0.0076(0.0000,0.0294) & 1 / 132 \\ 0.1000(0.0203,0.2304) & 3 / 30 \\ 0.0087(0.0000,0.0338) & 1 / 115 \\ 0.0176(\mathbf{0 . 0 0 2 4}, \mathbf{0 . 0 4 6 4 )} & \mathbf{5 / 3 0 6}\end{array}$

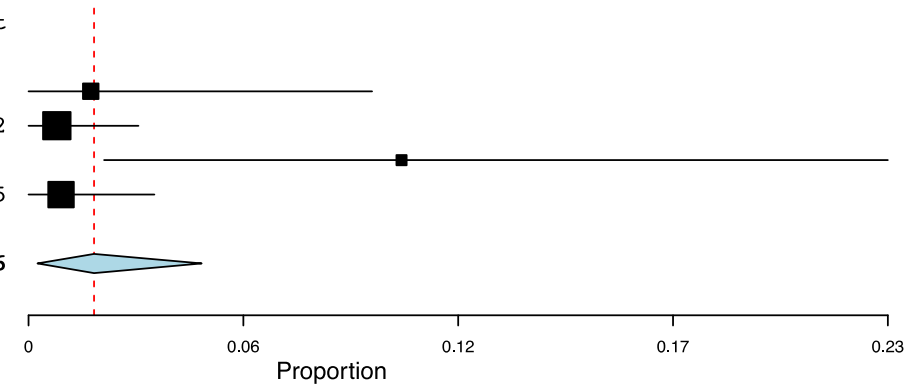

Studies

Propst 2020 erosion 3 mo

Thys 2020 erosion

Tam 2019 erosion 3 mo

Propst 2020 erosion 6 mo

Lone 2011 erosion

Tam 2019 erosion 6 mo

Cheung 2016 bleeding

Miceli 2020 erosion

Chien 2019 erosion

Overall $\left(I^{2}=76.3 \%, P<0.001\right)$

Fig. 3 Erosion meta-analysis
Estimate (95\% C.I.) Ev/Trt

$0.074(0.020,0.158)$

$0.004(0.000,0.021)$

$0.034(0.000,0.130)$

$0.018(0.000,0.067)$

$0.022(0.007,0.046)$

$0.200(0.079,0.359)$

$0.068(0.032,0.117)$

$0.070(0.030,0.123)$

$0.050(0.024,0.087) 43 / 865$

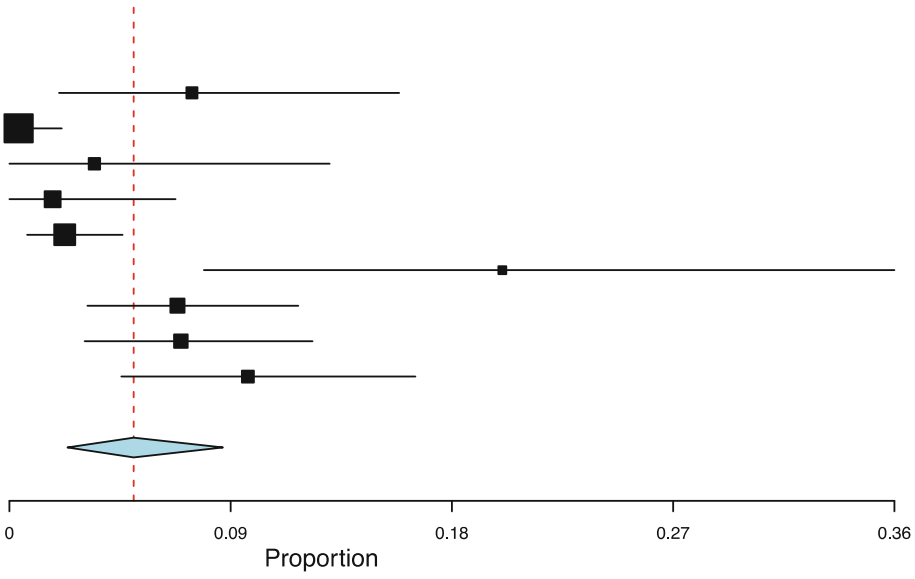

Studies

Tam 2019 retention 3 mo Tam 2019 retention 6 mo

Cheung 2016 retention

Chien 2019 retention

Overall $\left(I^{2}=56.2 \%, P=0.079\right)$

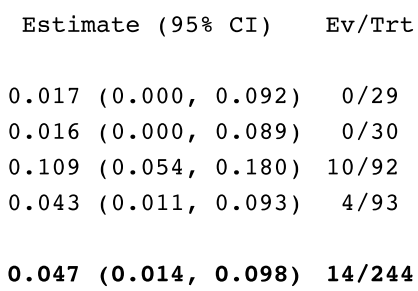

$0.047(0.014,0.098) 14 / 244$

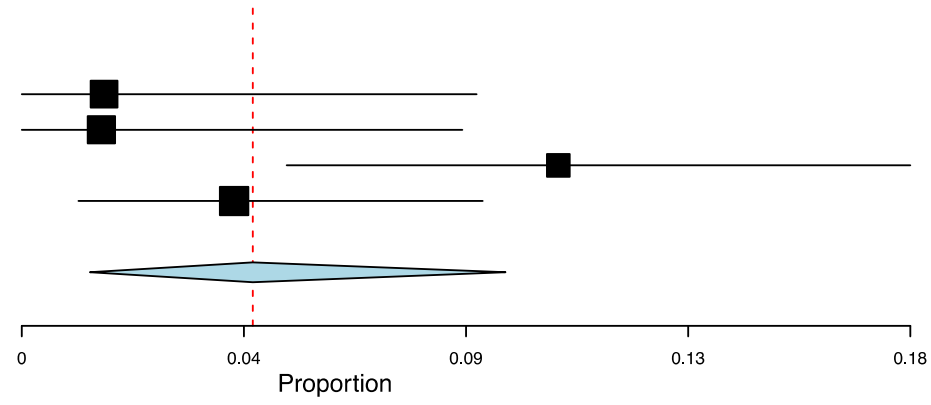

Fig. 4 Urinary retention meta-analysis 


\section{Empiric treatment of UTI}

Regarding management of recurrent UTIs, we sought articles on treatment of UTIs when urine cultures are not available; these included articles on whether to empirically treat with antibiotics, and if so, which antibiotic regimens to use. We sought to answer or include articles addressing the following questions: (1) one antibiotic versus another(s) for complicated or uncomplicated UTIs; (2) different durations of the same antibiotic for complicated or uncomplicated UTIs; (3) most appropriate treatment of patients with diabetes with UTI symptoms; (4) most appropriate treatment of patients with neurogenic bladder with UTI symptoms; (5) costeffectiveness of certain empiric management strategies; (6) predicting results of future culture from past culture(s); (7) predicting resistance or response to certain antibiotics based on patient characteristics; (8) risk factors for need for escalation of care (hospital admission and/or bacteremia) from community-acquired organism; (9) likelihood of a patient having a UTI based on symptomatology alone.

\section{Research question}

1. What is the best way to treat single-incident UTIs in older, more complicated patients without urine culture?

2. How should patients with known recurrent UTIs have UTI symptoms addressed if they cannot present for care?

\section{Study eligibility criteria (PICOS)}

\section{Population}

- Women with history of recurrent UTIs (per study definition)

- UTI in postmenopausal (or older) women with urogynecologic conditions (e.g., prolapse, incontinence)

\section{Intervention}

- Management of UTI without urine culture

\section{Comparator}

- None (single group studies)

- Other management plans without urine culture (e.g., different antibiotic regimen)

- Management with urine culture

\section{Outcomes}

- (Primary) emergency/urgent in-person care

- (Primary) adverse outcomes/complications
- (Secondary) clinical (patient-centered) outcomes

- (Secondary) receiving urogynecology care

Study design/article availability

- Primary studies of any design except case reports and case series

- Systematic review or guideline

- $\quad N \geq 10$ per intervention (group)

- English language publication

- Article immediately available for review

Literature search strategies Inception through March 30, 2020.

MEDLINE via PubMed.

("Clinical Decision-Making"[Mesh] OR "Clinical Decision Rules"[Mesh] OR "Decision Making”[Mesh] OR "Decision Support Techniques"[Mesh] OR Empiric or ((decisionmaking or decision-making) and clinical))

AND

("Urinary Tract Infections"[Mesh] or urinary tract infection* OR uti OR utis OR pyuria OR bacteriuria)

Limit to English.

Cochrane databases.

MeSH descriptor: [Clinical Decision-Making] OR ((decisionmaking or decision-making) and clinical) OR Empiric.

AND

MeSH descriptor: [Urinary Tract Infections] OR urinary tract infection OR uti OR utis OR pyuria OR bacteriuria OR urinary tract infections.

\section{Literature and screening Results}

The combined (partially deduplicated) searches yielded 1177 citations. These were screened singly in full by four team members. Among these, 116 citations were screened in, which were rescreened by a single team member, who selected 76 for further review of which 18 articles were not available in full text. In total, 60 articles provided information, which included 4 articles found within the searches for telemedicine or voiding dysfunction. These included 2 RCTs, 13 nonrandomized comparative studies, 7 single group studies, and other articles including consensus documents, costeffectiveness analyses, and narrative reviews. Pertinent articles are cited in the paper.

For studies on urinary tract infection, we extracted data elements on study design, study population, main antibiotic advocated for use as empiric therapy, treatment outcomes (\% not treated appropriately, \% susceptible to given antibiotic, worsening of symptoms), symptoms predictive of UTI, correspondence between urine cultures in bacterial strain and/or resistance profile, and cost of care calculations. 


\section{Voiding dysfunction and retention}

Regarding management of urinary voiding dysfunction (or urinary retention), we sought articles on risk of any adverse event associated with untreated urinary retention, management of self-catheterization (particularly related to virtual training of patients), use of prophylactic antibiotics in women not receiving surgery or other procedures, and virtual physical therapy, behavioral therapies, and simple devices to aid management, ways to remotely assess adequate bladder emptying, and pharmaceutical options to help improve bladder emptying.

\section{Research question}

1. In women with voiding dysfunction, what is the risk of adverse outcomes with untreated urinary retention?

\section{Study eligibility criteria (PICOS)}

\section{Population}

- Women with urinary dysfunction or retention

\section{Interventions}

- Virtual training in self-catheterization

- Prescribing antibiotics prophylactically

- Virtual assessment of adequate bladder emptying (i.e., voiding trials) and catheter management

- Medical treatment of urinary retention

- Virtual training in behavioral techniques/voiding strategies for more complete voiding

\section{Outcomes}

- Rates of adverse events associated with untreated urinary retention

- Success with remote teaching of clean intermittent selfcatheterization

- Rates of urinary tract infections associated with voiding dysfunction

- Ability of patients to adequately empty their bladders

- Success at determining remotely if a patient is adequately emptying her bladder

\section{Study design/article availability}

- Primary studies of any design except case reports and case series

- Not for prophylactic antibiotics
- Systematic review or guideline

- In particular for prophylactic antibiotics

- $\quad N \geq 10$ per intervention (group)

- English language publication

- Article immediately available for review

Literature search strategies Inception through March 31, 2020.

MEDLINE via PubMed.

((“Urinary Retention”[Mesh] OR Urinary retention OR

"Voiding dysfunction")

NOT

("Prostate"[Mesh] OR prostate OR "Prostatic Hyperplasia"[Mesh]))

AND

("Hydronephrosis"[Mesh] OR Hydronephros* OR "Intermittent Urethral Catheterization"[Mesh] OR "Urinary Catheterization"[Mesh] OR "Catheters, Indwelling"[Mesh] OR Self-Catheterization OR Foley OR.

Catheter OR Catheterization OR "Antibiotic Prophylaxis"[Mesh] OR “Anti-Bacterial Agents"[Mesh] OR.

((antibiotic OR antibiotics OR anti-bacterial) AND (prophylaxis OR prophylactic)) OR "Physical Therapy Modalities"[Mesh] OR "Physical therapy" OR "Physical therapies" OR "Physical therapeutic" OR Physiotherapy OR "Breathing Exercises"[Mesh] OR Breathing OR "Behavior Therapy"[Mesh] OR Crede OR "Pessaries"[Mesh] OR Pessar*)

NOT

("Male"[Mesh] NOT "Female”[Mesh])

Limit to English.

Cochrane databases.

((MeSH descriptor: [Urinary Retention] OR Urinary retention OR "Voiding dysfunction")

NOT

(MeSH descriptor: [Prostate] OR prostate))

AND

(MeSH descriptor: [Hydronephrosis] OR MeSH descriptor: [Intermittent Urethral Catheterization] OR MeSH descriptor: [Urinary Catheterization] OR MeSH descriptor: [Catheters, Indwelling] explode all trees OR MeSH descriptor: [Antibiotic Prophylaxis] OR MeSH descriptor: [AntiBacterial Agents] OR MeSH descriptor: [Physical Therapy Modalities] OR MeSH descriptor: [Breathing Exercises] OR MeSH descriptor: [Behavior Therapy] OR MeSH descriptor: [Pessaries] OR Hydronephros* OR Self-Catheterization OR Foley OR Catheter OR Catheterization OR ((antibiotic OR antibiotics OR anti-bacterial) AND (prophylaxis OR prophylactic)) OR "Physical therapy" OR "Physical therapies" OR "Physical therapeutic" OR Physiotherapy OR Breathing OR Crede OR Pessar*) 


\section{Literature and screening results}

The combined (partially deduplicated) searches yielded 2935 citations. These were screened singly by seven team members. Abstracts were screened until the Abstrackr program predicted that no remaining unscreened abstracts would be relevant (when all remaining prediction values were $<0.40$ ). Thus, first-round screening stopped after 2525 citations had been screened. In total, 152 citations were screened in, which were rescreened by a single team member, who selected 40 for further review, 5 of which were not available. After full-text screening, 10 articles were included for data extraction and 12 narrative reviews provided additional information (one of which was identified by a team member outside the literature review). All included articles are cited in the paper.

\section{Urinary incontinence}

Of note, as cited in the paper, several coauthors recently published a comprehensive systematic review for the Agency for Healthcare Research and Quality (AHRQ) Evidence-based Practice Center Program and the Patient Centered Outcome Research Institute (PCORI) on nonsurgical treatments for women with urinary incontinence (also published in the Annals of Internal Medicine and Journal of General Internal Medicine). The guidance on management of urinary incontinence was in part based on this review. Additional studies published since August 2018 were included. All included articles are cited in the paper.

\section{Appendix 2}

\section{Telemedicine in FPMRS patients}

A critical element of transitioning to telemedicine is maintaining the unique elements of trust, privacy and informationsharing that occur between provider and patient. A study comparing screen-to-screen and face-to-face interactions between FPMRS providers and patients with pelvic organ prolapse highlight three main communicative functions of a medical interaction: (1) information exchange, (2) relationship building, and (3) perceived shared decision-making. The authors concluded that virtual visits can provide similar patient satisfaction by building strong therapeutic relationships with patients through education, active listening, and shared decisionmaking [9]. These findings were confirmed in a rural extension project which focused on the impact of telemedicine on women with stress urinary incontinence who may have unique barriers to in-person visits. The authors found slightly improved adherence to follow-up visits and a 33\% acceptance of virtual visits in this previously telemedicine-naive population [12].
Interestingly, rural environments may also struggle with adequate internet availability, further confounding the problem of follow-up medical care. In addition, while these studies suggest successful integration of virtual visits in FPMRS, there is no validated questionnaire assessing patient satisfaction with telemedicine care.

As a surgical subspecialty, many FPMRS visits include short- and long-term postoperative assessments, and several groups have considered converting these to virtual visits. A feasibility study assessing reliability and patient satisfaction with telephone follow-up after either anterior colporrhaphy or midurethral sling showed a high concordance between telephone and in-office follow-up; over $90 \%$ of SUI patients and over $100 \%$ of POP patients were accurately evaluated by phone interview [4].

Two subgroups showed inadequate evaluation with the telephone interview. The first were sling patients reporting urinary incontinence who were ultimately diagnosed with de-novo urge incontinence rather than recurrent SUI. The second group was asymptomatic patients who were found to have a mesh erosion on physical examination. Previous studies showed that about $50 \%$ of patients following anterior colporrhaphy or midurethral sling could be followed telephonically, but this was confounded by the high rate of complex, combined surgical procedures and perioperative complications. Therefore, this study suggests that a more selective postoperative cohort may be best suited for telephone follow-up after surgery, specifically following MUS for patients without incontinence and following POP repair without mesh implantation. In the current COVID-19 healthcare crisis, there is growing pressure to reduce the volume of patients seen in person and to convert visits to telemedicine. Typically, surgeons feel compelled to see all postoperative patients, but these data suggest that we may be able to stratify patients by perioperative risk and decrease the total number of direct-care visits to enhance physical distancing. Even postoperative FPMRS patients derived from a high-volume, expert surgical practice who participated in telephone contact following pelvic surgery demonstrated high patient satisfaction and no difference in adverse events, ER, or PCP visits or clinical outcomes [10]. Located in the western US, this group suggested that implementation of alternative methods for delivering health care should incorporate considerations for household income, distance traveled, and need for accompaniment.

During the initial phase of community transmission of COVID-19, most FPMRS procedures will be (have been) canceled, so postoperative visits for these patients represent cases performed prior to changes in healthcare delivery. Transitioning to telemedicine for those patients may well protect them from unnecessary viral exposure. In the same way, as elective surgeries are slowly reintroduced and more urgent cases are prioritized, postoperative care can utilize virtual visits to prolong physical distancing. 
Telemedicine may be effectively used for providing a preliminary assessment of new patients and to assist in the follow-up of uncomplicated established patients [7]. In addition to physical distancing necessary for community-based COVID-19 management, virtual visits can reduce the carbon footprint and diminish interruption of vocational activities of patients and family members. Strategic pre-screening of patients for virtual visits by home completion of a symptom assessment provides an opportunity for FPMRS providers to triage eligible patients. Those with symptomatic prolapse will most likely request treatment in the form of a pessary or surgery, and those who elect pelvic floor physical therapy can receive guidance about the anticipated effectiveness based on the severity of their prolapse.

\section{Pessary management}

One randomized controlled trial stratified vaginal epithelial abnormalities into categories ranging from no epithelial abnormalities to an epithelial break or erosion of $>1 \mathrm{~cm}$ on a scale of 0 to 4 [18]. They reported use of a ring, gellhorn, or incontinence dish pessary with "routine follow up every 24 weeks is noninferior to every 12 weeks based on incidence of vaginal epithelial abnormalities." Specifically, the prevalence of grade 3 or 4 erosion was $7.4 \%$ for 3 -month cleanings and $1.7 \%$ for the extended 6 -month cleanings.

The study reporting the longest duration of continuous pessary use was a prospective cohort by Miceli et al. [17]. Subjects wore a pessary continuously for 2 years and were asked specifically not to remove, clean, or self-replace the pessary. Follow-up appointments were completed, but the pessary was not removed or cleaned at that time. The rate of bleeding or excoriation was $7.0 \%$. Additionally, $5.2 \%$ of women did report "feeling more vaginal discharge than is normal." However, only one culture $(0.9 \%)$ revealed abnormal pathogens (Candida glabrata) on culture.

A prospective cohort following 163 ring pessaries found no difference in pain, discharge, or irritation when the interval of cleaning was increased to 9 months. They also reported that $45.2 \%$ were able to manage self-cleaning for the directed period of time [21].

\section{Empiric treatment of UTI}

\section{Telehealth for UTI treatment}

In an RCT on telephone visits among uncomplicated patients with typical symptoms of UTI, telephone triage and management resulted in similar positive culture results and patient satisfaction compared with an in-office visit [5]. However, in this study, patients who participated in telephone triage were twice as likely to report persistent symptoms at 10 days, despite no difference in persistent bacteriuria. Virtual visits via "e-medicine" in a prospective cohort indicated that virtual visits for uncomplicated UTIs had lower cost and generated less laboratory work, but resulted in higher antibiotic prescribing [8]. Virtual visits for UTIs also generated less laboratory testing $(87.5 \%$ vs. $20.6 \%, p<0.001)$ and lower antibiotic fill rates within 3 days $(90.5 \%$ vs. $76.4 \%, p<0.001)$, with this retrospective study finding no difference in symptom resolution surveying a broad range of presenting infectious symptoms [6]. An expert opinion by Bent et al. [57] and review of several papers on symptom prediction of UTIs by DeAlleaume et al. [58] advocated for telephone-based therapy for patients with classic symptoms, with urine culture required only if atypical symptoms, citing that the presence of frequency and dysuria without vaginal symptoms gives women a $96 \%$ chance of having a UTI.

Common symptoms of UTIs, particularly in the absence of vaginal symptoms, are very predictive of UTIs, particularly in uncomplicated patients. The symptoms of dysuria $(81.1 \%$ UTI; +LR 1.23), urgency (98.4\% UTI; +LR 1.12), and burning with urination $(93.7 \%$ UTI; +LR 1.09) were advocated in a prospective cohort study as being significantly related to UTI presence $(p<0.05)$ [44]. The symptoms of dysuria (LR $1.3095 \%$ CI 1.20-1.41), frequency (LR $1.1095 \%$ CI 1.04 1.16), and hematuria (LR 1.72 95\% CI 1.30-2.27) were significantly predictive of UTI in a systematic review of 16 studies with 3711 patients [61].

It is noted by many authors that wide variation in UTI management is bad for patient health and antibiotic stewardship, and protocol-based therapy is an excellent way to prevent confusion and poor outcomes [59, 60]. A large European review of national guidelines of many countries found that significant variation in empiric therapy guidelines makes UTI therapy difficult to administer to wider populations [76], so wide adherence to evidence-based therapy or protocols for empiric treatment would be beneficial. For this reason, it is our expert opinion that institutions should base their treatment of choice, within the confines of first-line therapies recommended by guidelines such as those of 2010 from the Infectious Diseases Society of America (IDSA) and the European Society for Microbiology and Infectious Diseases (ESCMID) [84], on local antibiograms and upon what can be universally applied to their region and health system.

\section{Economic impact of empiric therapy strategies}

Regarding the economic impact of empiric therapy without urine laboratory testing, empiric treatment for 7 days was more cost-effective in women than all other treatment strategies [56], and a cost-minimization analysis found that empiric therapy with trimethoprim-sulfamethoxazole (TMP-SMZ) in elderly women was the most cost-effective strategy in these patients at a cost of $\$ 89.64 /$ patient [85]. Another study found that empiric treatment with TMP-SMZ was most cost- 
effective at the cost of $\$ 90.02$ per episode [86]. Empiric therapy as a strategy was less costly (14 pounds) than any strategy with laboratory testing, and laboratory testing costs 215 pounds per single day of avoided symptoms [87]. Regarding the cost of various antibiotics, Israeli national recommendations of nitrofurantoin use as an empiric antibiotic was found to be cost-effective ( $\$ 14.93 \pm \$ 8.18$ at a slightly higher cost than TMP-SMZ \$0.64 $\pm \$ 3.84$ and ofloxacin $\$ 2.75 \pm \$ 4.23$ ) in a retrospective cohort study [42]. Another cost-decision analysis found that nitrofurantoin was cost-effective when the prevalence of fluoroquinolone resistance exceeded $12 \%$ among uropathogens or the prevalence of TMP-SMX resistance exceeded $17 \%$ [88]. A cost/decision analysis of the strategy of fluoroquinolone use at various levels of TMP-SMZ resistance found that empiric use of fluoroquinolones was cost-effective when TMP-SMZ resistance in the community was 22\% (cost of fluoroquinolone $\$ 107$ and cost of TMPSMZ \$106 at this resistance level) [68]. For this reason, TMP-SMZ and nitrofurantoin seem most cost-effective, with nitrofurantoin being preferred in settings of TMP-SMZ resistance and fluoroquinolones reserved for when neither are appropriate.

\section{Predicting antibiotic resistance based on patient characteristics}

In predicting which patients may have antibiotic resistance from characteristics alone, one cross-sectional study found that women have no more resistance than men, indicating that resistance trends in local or national antibiograms can apply to a female population [63]. A prospective cohort on the role of $\mathrm{H} 30$ antigen in mismatched antibiotic therapy determined that age $>70$ ( $28 \%$ non-susceptible therapy), diabetes ( $30 \%$ nonsusceptible therapy), and catheterization (60\% nonsusceptible therapy) were associated with resistance of organisms [45]. A retrospective single-arm study of patients with uncomplicated and complicated UTIs hospitalized for community-acquired infections determined that cefazolin sensitivity was associated with age $<65$ and no diabetes, catheter use, or UTIs in last year [51]. A prospective cohort found that diabetes was a risk factor for failed therapy [64]. Another retrospective analysis of specifically FPMRS patients indicated that significant risk factors for needing to change antibiotics (an event that occurred in $26 \%$ of the population) from empiric therapy included immunosuppression [54]. A casecontrol study on patients with bacteremia versus nonbacteremic patients with community-acquired UTIs indicated that risk factors for bacteremia included a temperature $(+\mathrm{LR}$ 0.8620; X2 10.13) and diabetes mellitus (0.8110; X2 4.25), and risk factors for resistant organisms included advancing age and antibiotic exposure before admission [43]. For this reason, we suggest that factors associated with failure of first-line therapy and associated with greater illness be assessed thoroughly in all telemedicine visits regarding a possible UTI.

\section{Duration of empiric therapy}

In selecting optimal duration of therapy, expert narratives advocated for 3 days of antibiotics as opposed to 1 day dosing $[67,90]$ or as opposed to 7 days of therapy [143] for uncomplicated UTIs. For older patients, a 3-day (RR for efficacy versus single-dose $2.01,95 \%$ CI 1.05 to 3.84 ) or 7-day course of therapy (RR $1.93,1.01$ to $3.7095 \% \mathrm{CI}$ ) was more efficacious than single-dose therapy in a systematic review of 15 studies with 1644 elderly women [72]. For older patients, longer durations of therapy are also advocated by expert opinion [82]. It would appear that 3-7 days is appropriate for most patients, but that elderly women and/or nursing home patients should be treated on the upper end of this spectrum (7 days).

\section{Choice of empiric therapy}

Regarding choice of antibiotic for empiric therapy, one RCT in women with uncomplicated UTIs supported the use of norfloxacin $200 \mathrm{mg}$ BID for 5 days as opposed to ritapenam ( $2 \%$ failure therapy versus $6 \%, p=0.06$ ) [36], but neither arm of this study utilized therapies advised as first-line in guidelines. A prospective cohort study of use of fosfomycin $3 \mathrm{~g}$ orally in one dose demonstrated efficacy of this empiric strategy, with a $92.8 \%$ susceptibility and all patients having improvement in symptoms [50]. A regional study of European countries indicated that nitrofurantoin had the lowest resistance rate $(<5 \%)$ as opposed to penicillins (20-60\%), TMPSMZ (21-36\%), fluoroquinolones (19-40\%), and cephalosporins (2-17\%) [46]. An expert review from the infectious disease arena recommended fosfomycin or nitrofurantoin for empiric therapy based on this reasoning [62]. A second prospective study across the nation of Spain advocated cefixime, with a susceptibility of $99 \%$ with use as an empiric therapy, as opposed to augmentin, which was $<66 \%$ [38]. In contrast, in a large systematic review of studies that compared various antibiotics for uncomplicated UTIs, the meta-analyses supported the use of a fluoroquinolone (ciprofloxacin or gatifloxacin) based on these data [78], but these data did not include cost analyses or antibiotic stewardship concerns, merely efficacy. A single-arm prospective study of nationwide French antibiotic susceptibility suggested fosfomycin, pivmecillinam, and nitrofurantoin for uncomplicated cystitis, citing antimicrobial studies of susceptibility on all bacteria $(97.8 \%$ fosfomycin, 91.5\% nitrofurantoin, $98.3 \%$ ciprofloxacin; the lowest was ampicillin $61.4 \%$ ) and E. coli only (99\% fosfomycin, $97.1 \%$ pivmecillinam, $97.3 \%$ nitrofurantoin, $98.3 \%$ ciprofloxacin; the lowest was ampicillin at 61\%) [55]. This literature suggests that empiric therapy should be concurrent with the Infectious Diseases Society of America (IDSA) and 
European Society for Microbiology and Infectious Diseases (ESMID) guidelines of 2010 and involve the use of nitrofurantoin, TMP-SMZ, fosfomycin, or pivmecillinam (PMID 21292654)

\section{Patients with diabetes}

As noted above, diabetes is associated with more risk of failed therapy or resistance to antibiotics used empirically. Another prospective cohort of uncomplicated UTIs in mostly female patients advocated for nitrofurantoin, but noted that diabetes had a greater chance of failed therapy ( $44.4 \%$ versus $29.4 \%$ ) [64]. In fact, expert opinion advocates for more broadspectrum antibiotics (fluoroquinolones first line) in patients with diabetes, with use of Imipenem, ticarcillin-clavulanate, and piperacillin-tazobactam for seriously ill patients as Pseudomonas is more likely to be present [73].

\section{Reserved use of fluoroquinolones}

A retrospective cohort study of 274 ER patients indicated that empiric treatment with TMP-SMZ was inferior to empiric therapy to fluoroquinolones ( $16.4 \%$ vs. $34.4 \%$ ), but the authors still recommend TMP-SMZ as first line for antibiotic stewardship purposes [39], an approach advocated by expert authors on resistance emergence [79] and authors reviewing antibiograms city-wide in Seattle, WA, USA, where TMPSMZ had sensitivity of 95\% [40]. In fact, a case-control study of non-pregnant adults with UTI symptoms indicated that use of levofloxacin in the past was a significant risk factor for fluoroquinolone resistance $(2.0 ; 95 \% \mathrm{CI}, 1.0-3.9)$, as was each week of additional hospitalization (OR, 5.6; 95\% CI, 2.1-27.5), and they suggested use of TMP-SMZ as empiric therapy to avoid this resistance [41]. An empiric 3-day course of TMP-SMZ, with reservation of fluoroquinolones for patients with recurrent or complicated UTIs or contraindications to sulfa drugs, was also advocated by several expert reviews $[80,81,91]$. Narrative reviews also pointed to the 2010 Infectious Disease Society of America (IDSA) Guidelines (Pubmed 21,292,654) and, more recently, the 2017 EAU guidelines on urologic infections $[81,89]$. A systematic review summarizing uncomplicated UTI treatment advocated for recommendations very similar to the IDSA guidelines [77]. Both recommended that fluoroquinolones be advocated in special circumstances only, with first-line antibiotics being TMP-SMZ, nitrofurantoin, pivmecillinam, and fosfomycin.

\section{Choice of empiric therapy in nursing home populations}

A prospective cohort study specific to nursing home patients advocated TMP-SMZ or a first-generation cephalosporin at treatment of choice, with about $75 \%$ susceptibility for both [83]. A consensus statement by Delphi methods among experts advocated for nitrofurantoin in this population in case of normal renal function and TMP-SMZ with compromised renal function, with reservation of fluoroquinolones for patients with the poorest renal function [66]. Another nursing home population of a single-arm retrospective cohort advocated for TMP-SMZ for empiric therapy (86\% susceptible) and nitrofurantoin if culture suspected E. coli (87\% susceptible) [53]. An expert narrative review advocated for systematic fluoroquinolones (ofloxacin, lomefloxacin, or ciprofloxacin) as empiric therapy specifically in the nursing home population [71].

\section{Complicated infections}

A retrospective cohort of ED patients presenting with signs and symptoms of complicated infection supported the use of gentamicin or cephalosporins as empiric therapy if there were no risk factors (nursing home residence, hospitalization or antibiotic use within last 30 days, renal transplant, use of indwelling catheter, recurrent UTI history) and the use of a broad-spectrum beta lactam like piperacillin/tazobactam if risk factors were present [49]. For severe complicated UTIs, empiric therapy with ciprofloxacin $500 \mathrm{mg}$ bid, levofloxacin $500 \mathrm{mg}$ daily, or gatifloxacin $400 \mathrm{mg}$ daily [92] or treatment parenterally with a carbapenam or piperacillin/tazobactam [69] is advocated by expert opinion. A guidelines statement on complicated UTIs had similar recommendations, advocating for one of the following choices for parenteral therapy if hospitalization is deemed necessary: fluoroquinolones (ciprofloxacin and levo-floxacin), cephalosporins (ceftriaxone, ceftazidime, and cefipime), piperacillin/tazobactam, and carbapenems (ertapenem, imipenem, meropenem, and doripenem) [70].

\section{Predicting susceptibility by past culture results}

Prior urine culture results from patients were significantly correlated with subsequent urine culture results, indicating that empiric therapy could be based on past culture organism/sensitivity. In a retrospective cohort of 4351 patients in the US and Canada, in a setting where ciprofloxacin resistance was high (40\%; 95\% CI, 39.5\%-40.5\%), it was found that correspondence of a future culture to a prior culture was $57 \%$ [95\% CI, 55\%-59\% at 4-8 weeks between the cultures (at 32 weeks between cultures $49 \%$; $95 \%$ CI, 48\%-50\%)], whereas sensitivity to antibiotics was the same or better in $57 \%$ (95\% CI, $55 \%-59 \%)$, both of which were better $(p<0.05)$ compared with chance alone [48]. In this study, factors that predicted correspondence to a prior culture were less time between the two cultures, younger age, no antibiotics between cultures, no negative culture between, cultures obtained during the same admission, an outpatient setting, and an E. coli organism [48]. A retrospective cohort in Europe also found that resistance to 
ampicillin, trimethoprim, or ciprofloxacin is maintained for 3 months at least, while susceptibility to nitrofurantoin, ciprofloxacin, or trimethoprim in a previous sample supports their prescription for a re-infection for up to a year, and resistance to nitrofurantoin is low and short lived [62]. In cases of complicated UTIs, a retrospective cohort found that empiric treatment based on culture results in the last 6 months was an inappropriate choice only $23 \%$ of the time and empiric therapy based on no prior culture results was inappropriate in choice $67 \%$ of the time, where the odds ratio of accurate treatment was 6.9 (95\% CI 2.7 to 17.1) if prior culture data were used and 9.8 (95\% CI 2.2 to 43.1 ) if subsequent culture was the same species [47]. For neurogenic patients in a single-arm retrospective study [52], there was $56 \%$ correspondence with the same organism and for antibiotic sensitivity correspondence was $77.3 \%$ for ciprofloxacin, $78.5 \%$ for nitrofurantoin, and $75 \%$ for TMP-SMX. Therefore, we advocate that past cultures, if available between recently and up to 1 year ago, should be used to guide therapy chosen over telemedicine visits regarding UTIs.

\section{Recurrent UTI patients}

With recurrent UTI patients, it is notable that not getting cultures for each symptomatic episode is in contrast to recent recommendations by the American Urological Association (AUA), Canadian Urological Association (CUA), and Society of Urodynamics, Female Pelvic Medicine, and Urogenital Reconstruction (SUFU), which had grade C evidence to recommend this. However, this same set of guidelines had the same level of evidence for offering patientinitiated treatment when awaiting urine cultures (PMID 31042112) indicating that in this pandemic it may be reasonable to avoid the risk of exposure to healthcare contact for recurrent UTI patients and allow them to self-initiate empiric antibiotic therapy based on their prior cultures.

\section{Voiding dysfunction and retention}

\section{What is the risk of adverse events associated with untreated urinary retention?}

One of the best resources regarding the risks associated with non-neurogenic chronic urinary retention (CUR) was a white paper published by the American Urological Association (AUA) (28163030). However, this paper does not suggest ways in which physicians can remotely determine if someone with CUR (defined as an elevated PVR $>300 \mathrm{ml}$ that has persisted for at least 6 months and is documented on $\geq 2$ or more separate occasions) is at high risk for organ system harm or failure. They do suggest assessing which patients with CUR are high risk based on the following risk factors: imaging studies showing hydronephrosis, hydroureter and/or bladder stones; laboratory findings that indicate stage 3 chronic kidney disease (estimated glomerular filtration rate of 45 $49 \mathrm{ml} / \mathrm{min} / 1.73 \mathrm{~m}^{2}$ [stage $3 \mathrm{~A}$ ] or $30-44 \%$ [stage $3 \mathrm{~B}$ ]), and recurrent symptomatic UTIs (any pyelonephritis, $\geq 3$ UTIs in a 12-month period, and any episode of urosepsis in a 6month period). Thus, imaging and/or laboratory evaluation may be necessary in any patient with suspected CUR.

In a nested study within a non-blinded randomized controlled trial of two different voiding trial protocols, women who failed the voiding trial after urogynecologic surgery self-selected two management options: placement of indwelling or clean intermittent self-catheterization (CISC) (31121138). Women who chose CISC had more postoperative phone calls, but fewer postoperative office visits $(2 \pm 1 \mathrm{vs} .1 \pm$ $1 ; P<0.01)$; otherwise, outcomes were comparable including no difference in the rate of postoperative UTI. This is relevant to the COVID-19 pandemic, as surgeons may want to limit postoperative visits by suggesting CISC to these patients.

One retrospective review (CN-1451445) of same-day urogynecology surgery revealed that postoperative urinary retention (POUR) is not increased with spinal anesthesia. At this time when minimizing the potential aerosolization of the coronavirus, for instance, by avoiding intubation and extubation, this may be of interest to pelvic surgeons. Multivariate logistic regression demonstrated that age $<55$, diabetes, and cystocele $>$ stage 1 were risk factors for POUR. Type of anesthesia was not a risk factor.

\section{Are there ways to use telemedicine to remotely assess whether a patient is adequately emptying her bladder?}

We could not find any remote techniques for quantifiable assessment of post-void residual volume (PVR) in patients beyond having the patient perform self-catheterization after voiding.

However, in an ancillary analysis of an RCT of prophylactic antibiotics in 255 patients undergoing urogynecologic surgery, voiding trial was done by retrograde filling with $300 \mathrm{ml}$. When patients voided $\geq 200 \mathrm{cc}, 97 \%$ passed the void trial (95\% CI 93.5-99.3). These types of patients are very unlikely to need intervention for retention. If patients in this study voided $<100 \mathrm{ml}$ after backfilling, only $3 \%$ passed the void trial. Such patients should be sent home with either an indwelling catheter or be trained in CISC technique prior to discharge (29197314).

Another RCT of 150 women (mean age 59 years, 33\% underwent vaginal hysterectomy, $48 \%$ underwent anterior repair, and $75 \%$ underwent midurethral sling) randomized 75 patients $(50 \%)$ to a voiding trial (VT) in which the postvoid residual (PVR) was measured and $75(50 \%)$ to a PVR-free trial. There were no differences in baseline demographic or intraoperative characteristics between the two groups. The 
primary outcome, VT failure, was not significantly different (53\% PVR VT vs. 53\% PVR-free VT, $p=1.0$ ). There were no significant differences in days of postoperative catheterization $(1[0,4]$ in PVR VT vs. $1[0,4]$ in PVR-free VT, $p=0.90)$, UTI (20\% PVR VT vs. $20 \%$ PVR-free VT, $p=1.0$ ), or postoperative voiding dysfunction (4\% PVR VT vs. 5\% PVR-free $\mathrm{VT}, p=1.0$ ). The authors concluded that it is not necessary to check a postvoid residual following reconstructive pelvic surgery, and a patient can safely go home without catheterization as long as she is able to void $50 \%$ of the retrograde filled bladder volume [112].

One double-blinded RCT looked at women undergoing midurethral sling surgery [102]. The investigators found that women who voided at least $2 / 3$ of the instilled volume, or those that subjectively reported that the force of their urinary stream (FOS) was at least $50 \%$ of the strength it was prior to surgery (regardless of the voided volume), could be discharged without a catheter. Of the patients who were discharged home without a catheter in either group, none required catheter reinsertion within 6 weeks after the surgery. The subjective FOS could be particularly helpful when triaging patient complaints over the phone, as women who report $<50 \%$ FOS might need an in-person visit.

An RCT of 157 patients who underwent pelvic reconstructive surgery and failed a back-fill voiding trial in the hospital randomized 78 patients to self-discontinuation of the indwelling catheter on postoperative day (POD) 7 and 79 to office catheter discontinuation on POD 6-8. While there was a high rate of UTI in both arms of the study $(\sim 60 \%)$ in this protocol, self-discontinuation was non-inferior to office-based discontinuation and resulted in few patient encounters and improved patient experience [114].

\section{What techniques can be used in remote coaching of behavioral modification to facilitate better emptying and/or virtual training in self-catheterization?}

In a descriptive review, Burgio describes teaching voiding dysfunction patients how to relax their pelvic floor muscles in the clinic; she does not describe any remote teaching of this technique [94]. However, she does describe how patients can utilize relaxation skills outside the clinic setting. Her expert opinion is that behavioral treatment begins by encouraging the patient to create a relaxing environment and taking adequate time for voiding: "They are instructed to slow down, take a deep breath, relax their body, relax their pelvic floor muscles, and wait for the urine to flow...Anecdotally, some women have benefitted from double voiding, or lingering until another detrusor contraction brings about more complete emptying."

Another review article looking at voiding dysfunction in women discusses how to teach women CISC [95]. While it does not specifically discuss remote teaching, the techniques the authors describe could be applied to virtual training, especially if video conferencing is available: "The patient...is taught to use a clean technique and mirror; the patient lies down initially and inserts a fine-bore catheter. When proficient with the mirror, she is taught to insert the catheter by feel in the sitting or standing position."

One additional, excellent review addresses the differences between single, one-time use of a sterile catheter technique compared with reusable catheters for CISC. While there may certainly be instances where the former is advisable (i.e., immunosuppressed patients), there does not appear to be strong evidence that it is superior to CISC in the general population [98].

In an RCT of 85 women with postpartum retention, 40 patients were randomized to CISC and 45 patients to indwelling catheters. The median duration of catheterization was significantly shorter in the CISC group than in the indwelling catheter group (12 vs. $24 \mathrm{~h}, p<0.01$ ) [108].

In one scenario-based preference assessment study investigating the preference of POUR patients for CISC versus indwelling catheterization, 99\% preferred the option of CISC over indwelling catheterization when presented with data suggesting 2 days shorter catheterization and $20 \%$ reduced risk of UTI with CISC [104].

Two recent retrospective cohort studies investigating risk factors for failure to learn and adhere to CISC demonstrated that patients with obesity and a low Pencil and Paper test score (a 4-min test that includes exercises of grip, access to perineum, and cognitive tasks to explore the global capacity for the patient to self-catheterize) are less likely to be good candidates for CISC [105, 106]. Age $\geq 65$ was not predictive of failure. However, another study regarding this topic concluded that increasing age was the only variable identified on multivariate logistic regression as a risk factor for failure to learn CISC [110].

In another study of 154 patients (105 of whom were women) who performed CISC for a mean of 5 years, $80 \%$ felt that CISC was easy or very easy and $90 \%$ reported no or minimal pain while performing CISC. Multivariable analysis showed that severe pain with CISC was the only factor that predicted poor quality of life in this population [107].

We have included here a link to a video on instruction of CISC [117], which may be helpful to some providers in teaching their patients.

\section{What is the value of prophylactic antibiotics for patients with acute urinary retention to prevent infection and are there medical therapies to improve emptying?}

In a 2009 non-clinical, decision-analysis study looking at the risks and benefits of prophylactic antibiotics during CISC for voiding dysfunction after prolapse/incontinence surgery, the authors completed two models [101]. Under baseline 
assumptions, prophylactic antibiotics were favored in both models. In the true UTI model, prophylactic antibiotics had an $83 \%$ probability of no UTI or adverse events, which is $16 \%$ better than no prophylactic antibiotics. The authors concluded that prophylactic antibiotics are favored for prevention of UTI during CISC after prolapse/incontinence surgery.

A Cochrane review of 6 randomized controlled trials including 789 men and women evaluated the use of antibiotic therapy during short-term catheter use. Bacteriuria was used as a surrogate for a UTI in these studies [97]. Data from this review suggest that the use of an antibiotic treatment during catheter placement, or for the duration of catheter use, resulted in lower rates of bacteriuria. However, the studies reviewed in this analysis looking at symptomatic infection were conducted prior to 1986, at which time prophylactic intravenous antibiotics were not commonly used preoperatively, and were not investigating women with POUR after reconstructive pelvic surgery.

In a multicenter RCT of 151 participants with POUR after pelvic surgery, 75 were sent home with nitrofurantoin and 76 with placebo [96]. Demographics were similar between groups. The indication for surgery was prolapse (46\%), incontinence $(20 \%)$, or both (34\%). Participants were discharged with an indwelling catheter (58\%) or self-catheterization $(42 \%)$. Median duration of catheter use was 4 days (interquartile range 3-7). Thirteen women in the nitrofurantoin group and 13 women in the placebo group experienced urinary tract infection $(17.3 \%$ vs. $17.1 \%$, P5.97, relative risk [RR] [95\% CI] 1.01 [0.50-2.04]). Adverse symptoms possibly attributable to nitrofurantoin were common in both groups (68\% vs. $61 \%, p=0.34$, RR [95\% CI] 1.12 [0.88-1.43]). Daily nitrofurantoin did not reduce the incidence of culture-proven urinary tract infection among women with catheter-managed urinary retention after pelvic reconstructive surgery. Cultureconfirmed urinary tract infection was found in only half of symptomatic episodes. This study suggests that there is minimal value of prophylactic antibiotic use in women being catheterized for postoperative urinary retention.

A 2013 Cochrane review of "Drugs for treatment of urinary retention after surgery in adults" [93] included analysis of seven studies including 494 participants. Drug treatments assessed included cholinergic agents, alpha-blockers, sedatives, and prostaglandin. The only remote therapy that showed a statistically significant association with improved likelihood of spontaneous voiding compared with placebo (RR 1.39, 95\% CI 1.07 to 1.82 ) was a combination of cholinergic agents combined with sedative. However, there was significant heterogeneity in the two studies in this analysis, and only one of the two treatments could be given remotely. The outcome was not statistically different between the three arms in this individual study. Thus, there is no evidence from this review that orally administered drugs successfully treat urinary retention after surgery. This conclusion is consistent with that of another review article that clearly states that alpha-blockers, specifically, are not superior to placebo in the treatment of acute urinary retention in women [99].

However, an RCT published in early 2020 including 130 patients with POUR after anterior colporrhaphy randomized 65 patients to $0.4 \mathrm{mg}$ tamsulosin (an alpha antagonist) at bedtime versus 65 placebo controls (30984702). At 24 h postoperatively, various assessments were made using subjective criteria. There were mixed results in regard to the efficacy compared to placebo, and no primary outcome was defined. Tamsulosin was not more effective than placebo with respect to the frequency of urinary retention $(78.5 \%$ vs. $63.1 \%, p=$ 0.054). Feeling of incomplete bladder emptying was higher in the tamsulosin group $(76.9 \%$ vs. $63.1 \%)$, and the incidence of urinary retention was significantly lower in the tamsulosin group ( $20 \%$ vs. $36.9 \%)$, as was residual bladder volume and diameter of urinary flow.

In 2010 the Infectious Diseases Society of America (IDSA) published guidelines on the diagnosis, prevention, and treatment of catheter associated (CA) urinary tract infection (UTI) in adults [115]. The guidelines pertain to patients who are managed with indwelling catheterization (including short- $[<$ 30 day] and long-term [ $>30$ day] catheterization), intermittent catheterization, and condom catheterization. This paper includes 47 guidelines, but we found 2 that we felt were particularly pertinent to our review. The first is guideline 39, "Systemic antimicrobial prophylaxis should not be routinely used in patients with short-term or long-term catheterization, including patients who undergo surgical procedures, to reduce CA-asymptomatic bacteriuria (CA-ASB) or CA-UTI because of concern about selection of antimicrobial resistance." The second is guideline 39, "Data are insufficient to make a recommendation as to whether routine catheter change (e.g., every 2-4 weeks) in patients with functional long-term indwelling urethral or supra- pubic catheters reduces the risk of CAASB or CA-UTI, even in patients who experience repeated early catheter blockage from encrustation."

\section{Urinary incontinence}

\section{Stress urinary incontinence}

Behavioral therapy including bladder training, pelvic floor physical therapy or Kegel pelvic floor muscle exercises, weight loss, and yoga have demonstrated significant improvement and/or complete resolution of SUI symptoms. This is particularly advantageous in the current situation as patients can implement behavioral interventions without leaving home. Additional options include use of $\alpha$-agonists, behavioral therapy combined with estrogen, or estrogen therapy alone. It is also important to note that use of an $\alpha$-agonist, such as duloxetine, is considered off-label use in the UStates but is an approved indication in Europe. Incontinence pessaries are a 
common treatment option for SUI, but there were insufficient data for evaluation in the systematic review; likewise, data were lacking regarding a patient-fitted device (Uresta) or use of an incontinence tampon (Impressa), although the overall risk should be low and may be an option for patients while avoiding in-person clinic visits [119, 124].

\section{Urgency urinary incontinence}

Behavioral therapy was more effective than anticholinergics for cure or improvement of UUI; anticholinergic medications were also effective either alone or in combination with behavioral therapy [118]. There is emerging evidence that anticholinergic use may increase individual risk for dementia later in life [120]; however, as the risk is related to the dose and duration of use, and balancing the risk of exposure to COVID-19 versus short-term anticholinergic use, it would seem reasonable to use anticholinergic medications to avoid clinic visits. The data on the comparative efficacy of a $\beta_{3}$-adrenoceptor agonist are evolving, but this different drug class has less or no dementia risk compared with anticholinergics. Hypertension is one of the concerns for this class of medication; however, two recent systematic reviews did not show any increased risk of hypertension over placebo [121, 122]. Pharmaceuticals can be obtained by mail order or could be coordinated for pick up at a grocery store or drive-through pharmacy.

\section{Mixed urinary incontinence}

The 2019 systematic review did not find sufficient data specific to guide MUI treatment specifically, but, pragmatically, MUI treatment would constitute a combination of the aforementioned SUI and UUI treatments.

Kegels can be utilized for various types of urinary issues, these can be described over the phone but some patients may benefit from technology to help guide and track their pelvic floor kegels. A recent study evaluated both free and paid smartphone apps. There are many available options; a recent review found that Kegel Trainer was the highest ranked free app and Kegel Trainer Pro® the highest ranked paid app [123]. These apps can also be used as a treatment for prolapse.

\section{References}

1. Smith AC, Thomas E, Snoswell CL, Haydon H, Mehrotra A, Clemensen J, Caffery LJ (2020) Telehealth for global emergencies: Implications for coronavirus disease 2019 (COVID-19). J Telemed Telecare 1357633X20916567. https://doi.org/10.1177/ 1357633 X20916567.

2. Rogers RG, Swift S. The world is upside down; how coronavirus changes the way we care for our patients. Int Urogynecol J. 2020. https://doi.org/10.1007/s00192-020-04292-7.
3. Agency for Healthcare Research and Quality Evidence-based Practice Center Program Rapid Review Guidance Document. https://www.ahrq.gov/sites/default/files/wysiwyg/funding/ contracts/epc-vi/22-rapid_evidence_products_guidance.pdf. Accessed 4 Apr 2020.

4. Balzarro M, Rubilotta E, Trabacchin N, Mancini V, Costantini E, Artibani W, et al. A prospective comparative study of the feasibility and reliability of telephone follow-up in female urology: the patient Home Office novel evaluation (PHONE) study. Urology. 2020;136:82-7. https://doi.org/10.1016/j.urology.2019.10.021.

5. Barry HC, Hickner J, Ebell MH, Ettenhofer T. A randomized controlled trial of telephone management of suspected urinary tract infections in women. J Fam Pract. 2001;50:589-94.

6. Gordon AS, Adamson WC, DeVries AR. Virtual visits for acute, nonurgent care: A claims analysis of episode-level utilization. J Med Internet Res. 2017;19:e35. https://doi.org/10.2196/jmir. 6783.

7. Jones G, Brennan V, Jacques R, Wood H, Dixon S, Radley S. Evaluating the impact of a "virtual clinic" on patient experience, personal and provider costs of care in urinary incontinence: A randomised controlled trial. PLoS One. 2018;13:e0189174. https://doi.org/10.1371/journal.pone.0189174.

8. Mehrotra A, Paone S, Martich GD, Albert SM, Shevchik GJ. A comparison of care at e-visits and physician office visits for sinusitis and urinary tract infection. JAMA Intern Med. 2013;173:72-4. https://doi.org/10.1001/2013.jamainternmed.305.

9. Tates K, Antheunis ML, Kanters S, Nieboer TE, Gerritse MB. The effect of screen-to-screen versus face-to-face consultation on doctor-patient communication: an experimental study with simulated patients. J Med Internet Res. 2017;19:e421. https://doi.org/ 10.2196/jmir.8033.

10. Thompson JC, Cichowski SB, Rogers RG, Qeadan F, Zambrano J, Wenzl C, et al. Outpatient visits versus telephone interviews for postoperative care: a randomized controlled trial. Int Urogynecol J. 2019;30:1639-46. https://doi.org/10.1007/s00192-019-03895$\mathrm{z}$.

11. Akbar N, Dobson EL, Keefer M, Munsiff S, Dumyati G. 1082. Hold the phone: antibiotic prescribing practices associated with nonvisit encounters for urinary tract infections (UTIs) in urology clinics. Open Forum Infect Dis. 2019;6:S384. https://doi.org/10. 1093/ofid/ofz360.946.

12. Schlittenhardt M, Smith SC, Ward-Smith P. Tele-continence care: A novel approach for providers. Urol Nurs. 2016;36:217-23.

13. International Urogynecologic Association (IUGA) Your Pelvic Floor Leaflets. https://www.yourpelvicfloor.org/leaflets/. Accessed 5 Apr 2020.

14. American Urogynecologic Association (AUGS) Voices for Pelvic Floor Disorders Fact Sheets and Downloads. https://www. voicesforpfd.org/resources/fact-sheets-and-downloads/. Accessed 5 Apr 2020.

15. National Association for Continence (NAFC) Urinary incontinence Education Learning Library. https://www.nafc.org/ learning-library. Accessed 5 Apr 2020.

16. Centers for Medicare and Medicaid Services (CMS) Medicare Telemedicine Healthcare Provider Fact Sheet. https://www.cms. gov/newsroom/fact-sheets/medicare-telemedicine-health-careprovider-fact-sheet. Accessed 3 Apr 2020.

17. Miceli A, Fernández-Sánchez M, Polo-Padillo J, Dueñas-Díez JL. Is it safe and effective to maintain the vaginal pessary without removing it for 2 consecutive years? Int Urogynecol J. 2020. https://doi.org/10.1007/s00192-020-04240-5.

18. Propst K, Mellen C, O'Sullivan DM, Tulikangas PK. Timing of office-based pessary care: A randomized controlled trial. Obstet Gynecol. 2020;135:100-5. https://doi.org/10.1097/AOG. 0000000000003580 . 
19. Tam M-S, Lee VYT, Yu ELM, Wan RSF, Tang JSM, He JMY, et al. The effect of time interval of vaginal ring pessary replacement for pelvic organ prolapse on complications and patient satisfaction: A randomised controlled trial. Maturitas. 2019;128:29 35. https://doi.org/10.1016/j.maturitas.2019.07.002.

20. Cheung RYK, Lee JHS, Lee LL, Chung TKH, Chan SSC. Vaginal pessary in women with symptomatic pelvic organ prolapse: A randomized controlled trial. Obstet Gynecol. 2016;128:73-80. https://doi.org/10.1097/AOG.0000000000001489.

21. Thys SD, Hakvoort RA, Asseler J, Milani AL, Vollebregt A, Roovers JP. Effect of pessary cleaning and optimal time interval for follow-up: a prospective cohort study. Int Urogynecol J. 2020. https://doi.org/10.1007/s00192-019-04200-8.

22. Chien C-W, Lo T-S, Tseng L-H, Lin Y-H, Hsieh W-C, Lee S-J. Long-term outcomes of self-management Gellhorn pessary for symptomatic pelvic organ prolapse. Female Pelvic Med Reconstr Surg. 2019. https://doi.org/10.1097/SPV. 0000000000000770

23. Lone F, Thakar R, Sultan AH, Karamalis G. A 5-year prospective study of vaginal pessary use for pelvic organ prolapse. Int $\mathrm{J}$ Gynaecol Obstet. 2011;114:56-9. https://doi.org/10.1016/j.ijgo. 2011.02.006

24. Wu V, Farrell SA, Baskett TF, Flowerdew G. A simplified protocol for pessary management. Obstet Gynecol. 1997;90:990-4. https://doi.org/10.1016/s0029-7844(97)00481-x.

25. Coolen A-LWM, Troost S, Mol BWJ, Roovers J-PWR, Bongers MY. Primary treatment of pelvic organ prolapse: pessary use versus prolapse surgery. Int Urogynecol J. 2018;29:99-107. https:// doi.org/10.1007/s00192-017-3372-x.

26. Yang J, Han J, Zhu F, Wang Y. Ring and Gellhorn pessaries used in patients with pelvic organ prolapse: a retrospective study of 8 years. Arch Gynecol Obstet. 2018;298:623-9. https://doi.org/10. 1007/s00404-018-4844-z.

27. Ramsay S, Tu LM, Tannenbaum C. Natural history of pessary use in women aged $65-74$ versus 75 years and older with pelvic organ prolapse: a 12-year study. Int Urogynecol J. 2016;27:1201-7. https://doi.org/10.1007/s00192-016-2970-3.

28. Collins S, Beigi R, Mellen C, O'Sullivan D, Tulikangas P. The effect of pessaries on the vaginal microenvironment. Am J Obstet Gynecol. 2015;212:60.e1-6. https://doi.org/10.1016/j.ajog.2014. 07.024 .

29. de A Coelho SC, Giraldo PC, Florentino JO, de Castro EB, LGO B, CRT J. Can the pessary use modify the vaginal microbiological flora? A cross-sectional study. Rev Bras Ginecol Obstet. 2017;39: 169-74. https://doi.org/10.1055/s-0037-1601437.

30. Deng M, Ding J, Ai F, Zhu L. Clinical use of ring with support pessary for advanced pelvic organ prolapse and predictors of its short-term successful use. Menopause. 2017;24:954-8. https:// doi.org/10.1097/GME.0000000000000859.

31. Manchana T. Ring pessary for all pelvic organ prolapse. Arch Gynecol Obstet. 2011;284:391-5. https://doi.org/10.1007/ s00404-010-1675-y.

32. Dessie SG, Armstrong K, Modest AM, Hacker MR, Hota LS. Effect of vaginal estrogen on pessary use. Int Urogynecol J. 2016;27:1423-9. https://doi.org/10.1007/s00192-016-3000-1.

33. Deng M, Ding J, Ai F, Zhu L. Successful use of the Gellhorn pessary as a second-line pessary in women with advanced pelvic organ prolapse. Menopause. 2017;24:1277-81. https://doi.org/10. 1097/GME.0000000000000909.

34. Sasaki T, Agari T, Date I. Devices and practices for improving the accuracy of deep brain stimulation. No Shinkei Geka. 2018;46: 751-62. https://doi.org/10.11477/mf.1436203809.

35. Kasper S. Editorial issue 4/2019. Int J Psychiatry Clin Pract. 2019;23:237. https://doi.org/10.1080/13651501.2019.1688484.

36. Swedish Urinary Tract Infection Study Group. Interpretation of the bacteriologic outcome of antibiotic treatment for uncomplicated cystitis: impact of the definition of significant bacteriuria in a comparison of ritipenem acoxil with norfloxacin. Swedish urinary tract infection study group. Clin Infect Dis. 1995;20:507-13.

37. Jansåker F, Frimodt-Møller N, Bjerrum L, Dahl Knudsen J. The efficacy of pivmecillinam: 3 days or 5 days t.i.d against community acquired uncomplicated lower urinary tract infections - a randomized, double-blinded, placebo-controlled clinical trial study protocol. BMC Infect Dis. 2016;16:727. https://doi.org/10.1186/ s12879-016-2022-0.

38. García-Rodríguez JA. Bacteriological comparison of cefixime in patients with noncomplicated urinary tract infection in Spain. Preliminary Results Chemotherapy. 1998;44(Suppl 1):28-30. https://doi.org/10.1159/000048461.

39. Guneysel O, Onur O, Erdede M, Denizbasi A. Trimethoprim/ sulfamethoxazole resistance in urinary tract infections. J Emerg Med. 2009;36:338-41. https://doi.org/10.1016/j.jemermed.2007. 08.068 .

40. Johnson JR, Stamm WE. Urinary tract infections in women: diagnosis and treatment. Ann Intern Med. 1989;111:906-17. https:// doi.org/10.7326/0003-4819-111-11-906.

41. Johnson L, Sabel A, Burman WJ, Everhart RM, Rome M, MacKenzie TD, et al. Emergence of fluoroquinolone resistance in outpatient urinary Escherichia coli isolates. Am J Med. 2008;121:876-84. https://doi.org/10.1016/j.amjmed.2008.04. 039.

42. Kahan NR, Kahan E, Waitman D-A, Chinitz DP. Economic evaluation of an updated guideline for the empiric treatment of uncomplicated urinary tract infection in women. Isr Med Assoc J. 2004;6:588-91.

43. Leibovici L, Greenshtain S, Cohen O, Wysenbeek AJ. Toward improved empiric management of moderate to severe urinary tract infections. Arch Intern Med. 1992;152:2481-6.

44. Mishra B, Srivastava S, Singh K, Pandey A, Agarwal J. Symptombased diagnosis of urinary tract infection in women: are we overprescribing antibiotics? Int J Clin Pract. 2012;66:493-8. https:// doi.org/10.1111/j.1742-1241.2012.02906.x.

45. Tchesnokova V, Riddell K, Scholes D, Johnson JR, Sokurenko EV. The uropathogenic Escherichia coli subclone sequence type 131-H30 is responsible for most antibiotic prescription errors at an urgent care clinic. Clin Infect Dis. 2019;68:781-7. https://doi.org/ 10.1093/cid/ciy523.

46. van der Donk CFM, van de Bovenkamp JHB, De Brauwer EIGB, De Mol P, Feldhoff K-H, Kalka-Moll WM, et al. Antimicrobial resistance and spread of multi drug resistant Escherichia coli isolates collected from nine urology services in the Euregion MeuseRhine. PLoS One. 2012;7:e47707. https://doi.org/10.1371/ journal.pone.0047707.

47. Linsenmeyer K, Strymish J, Gupta K. Two simple rules for improving the accuracy of empiric treatment of multidrug-resistant urinary tract infections. Antimicrob Agents Chemother. 2015;59: 7593-6. https://doi.org/10.1128/AAC.01638-15.

48. MacFadden DR, Ridgway JP, Robicsek A, Elligsen M, Daneman N. Predictive utility of prior positive urine cultures. Clin Infect Dis. 2014;59:1265-71. https://doi.org/10.1093/cid/ciu588.

49. Bischoff S, Walter T, Gerigk M, Ebert M, Vogelmann R. Empiric antibiotic therapy in urinary tract infection in patients with risk factors for antibiotic resistance in a German emergency department. BMC Infect Dis. 2018;18:56. https://doi.org/10.1186/ s12879-018-2960-9.

50. Bonfiglio G, Mattina R, Lanzafame A, Cammarata E, Tempera G, Italian Medici Medicina Generale (MMG) Group. Fosfomycin tromethamine in uncomplicated urinary tract infections: a clinical study. Chemotherapy. 2005;51:162-6. https://doi.org/10.1159/ 000085625 . 
51. Chiu C-C, Lin T-C, Wu R-X, Yang Y-S, Hsiao P-J, Lee Y, et al. Etiologies of community-onset urinary tract infections requiring hospitalization and antimicrobial susceptibilities of causative microorganisms. J Microbiol Immunol Infect. 2017;50:879-85. https://doi.org/10.1016/j.jmii.2016.08.008.

52. Clark R, Welk B. The ability of prior urinary cultures results to predict future culture results in neurogenic bladder patients. Neurourol Urodyn. 2018;37:2645-50. https://doi.org/10.1002/ nau. 23713

53. Datta R, Advani S, Rink A, Bianco L, Van Ness PH, Quagliarello $\mathrm{V}$, et al. Increased fluoroquinolone-susceptibility and preserved nitrofurantoin-susceptibility among Escherichia coli urine isolates from women long-term care residents: A brief report. Open Access J Gerontol Geriatr Med. 2018. https://doi.org/10.19080/ OAJGGM.2018.04.555636.

54. Dokter J, Tennyson LE, Nguyen L, Han E, Sirls LT. The clinical rate of antibiotic change following empiric treatment for suspected urinary tract infections. Int Urol Nephrol. 2020;52:431-6. https:// doi.org/10.1007/s11255-019-02327-7.

55. Neuzillet Y, Naber KG, Schito G, Gualco L, Botto H. French results of the ARESC study: clinical aspects and epidemiology of antimicrobial resistance in female patients with cystitis. Implications for empiric therapy. Med Mal Infect. 2012;42:6675. https://doi.org/10.1016/j.medmal.2011.07.005.

56. Barry HC, Ebell MH, Hickner J. Evaluation of suspected urinary tract infection in ambulatory women: a cost-utility analysis of office-based strategies. J Fam Pract. 1997;44:49-60.

57. Bent $\mathrm{S}$, Saint $\mathrm{S}$. The optimal use of diagnostic testing in women with acute uncomplicated cystitis. Am J Med. 2002;113(Suppl 1A):20S-8S. https://doi.org/10.1016/s0002-9343(02)01056-2.

58. DeAlleaume L, Tweed EM, Bonacci R. Clinical inquiries. When are empiric antibiotics appropriate for urinary tract infection symptoms? J Fam Pract. 2006;55(338):341-2.

59. Ross AM. UTI antimicrobial resistance: tricky decisions ahead? Br J Gen Pract. 2000;50:612-3.

60. Dixon T. Urinary tract infections. Management mayhem? Can Fam Physician. 1993;39:474-9.

61. Giesen LGM, Cousins G, Dimitrov BD, van de Laar FA, Fahey T. Predicting acute uncomplicated urinary tract infection in women: a systematic review of the diagnostic accuracy of symptoms and signs. BMC Fam Pract. 2010;11:78. https://doi.org/10.1186/ 1471-2296-11-78.

62. Vellinga A, Cormican M, Hanahoe B, Murphy AW. Predictive value of antimicrobial susceptibility from previous urinary tract infection in the treatment of re-infection. Br J Gen Pract. 2010;60: 511-3. https://doi.org/10.3399/bjgp10X514765.

63. McGregor JC, Elman MR, Bearden DT, Smith DH. Sex- and agespecific trends in antibiotic resistance patterns of Escherichia coli urinary isolates from outpatients. BMC Fam Pract. 2013;14:25. https://doi.org/10.1186/1471-2296-14-25.

64. George CE, Norman G, Ramana GV, Mukherjee D, Rao T. Treatment of uncomplicated symptomatic urinary tract infections: resistance patterns and misuse of antibiotics. J Family Med Prim Care. 2015;4:416-21. https://doi.org/10.4103/2249-4863. 161342.

65. Anger J, Lee U, Ackerman AL, Chou R, Chughtai B, Clemens JQ, et al. Recurrent uncomplicated urinary tract infections in women: AUA/CUA/SUFU guideline. J Urol. 2019;202:282-9. https://doi. org/10.1097/JU.0000000000000296.

66. Hanlon JT, Perera S, Drinka PJ, Crnich CJ, Schweon SJ, KleinFedyshin M, et al. The IOU consensus recommendations for empirical therapy of cystitis in nursing home residents. J Am Geriatr Soc. 2019;67:539-45. https://doi.org/10.1111/jgs.15726.

67. Andriole VT. When to do culture in urinary tract infections. Int $\mathbf{J}$ Antimicrob Agents. 1999;11:253-5; discussion 261. https://doi. org/10.1016/s0924-8579(99)00025-4.
68. Le TP, Miller LG. Empirical therapy for uncomplicated urinary tract infections in an era of increasing antimicrobial resistance: a decision and cost analysis. Clin Infect Dis. 2001;33:615-21. https://doi.org/10.1086/322603.

69. Bader MS, Hawboldt J, Brooks A. Management of complicated urinary tract infections in the era of antimicrobial resistance. Postgrad Med. 2010;122:7-15. https://doi.org/10.3810/pgm. 2010.11.2217.

70. Hsueh P-R, Hoban DJ, Carmeli Y, Chen S-Y, Desikan S, Alejandria $\mathrm{M}$, et al. Consensus review of the epidemiology and appropriate antimicrobial therapy of complicated urinary tract infections in Asia-Pacific region. J Inf Secur. 2011;63:114-23. https://doi.org/10.1016/j.jinf.2011.05.015.

71. McCue JD. Rationale for the use of oral fluoroquinolones as empiric treatment of nursing home infections. Arch Fam Med. 1994;3:157-64.

72. Lutters M, Vogt-Ferrier NB (2008) Antibiotic duration for treating uncomplicated, symptomatic lower urinary tract infections in elderly women. Cochrane Database Syst Rev CD001535. https:// doi.org/10.1002/14651858.CD001535.pub2.

73. Stapleton A. Urinary tract infections in patients with diabetes. Am J Med. 2002;113(Suppl 1A):80S-4S. https://doi.org/10.1016/ s0002-9343(02)01062-8.

74. Wojno KJ, Baunoch D, Luke N, Opel M, Korman H, Kelly C, et al. Multiplex PCR based urinary tract infection (UTI) analysis compared to traditional urine culture in identifying significant pathogens in symptomatic patients. Urology. 2020;136:119-26. https://doi.org/10.1016/j.urology.2019.10.018.

75. Kranjčec B, Papeš D, Altarac S. D-mannose powder for prophylaxis of recurrent urinary tract infections in women: a randomized clinical trial. World J Urol. 2014;32:79-84. https://doi.org/10. 1007/s00345-013-1091-6.

76. McQuiston Haslund J, Rosborg Dinesen M, Sternhagen Nielsen $\mathrm{AB}$, Llor C, Bjerrum L. Different recommendations for empiric first-choice antibiotic treatment of uncomplicated urinary tract infections in Europe. Scand J Prim Health Care. 2013;31:235-40. https://doi.org/10.3109/02813432.2013.844410.

77. Naber KG, Wullt B, Wagenlehner FME. Antibiotic treatment of uncomplicated urinary tract infection in premenopausal women. Int J Antimicrob Agents. 2011;38(Suppl):21-35. https://doi.org/ 10.1016/j.ijantimicag.2011.09.003.

78. Knottnerus BJ, Grigoryan L, Geerlings SE, Moll van Charante EP, Verheij TJM, Kessels AGH, et al. Comparative effectiveness of antibiotics for uncomplicated urinary tract infections: network meta-analysis of randomized trials. Fam Pract. 2012;29:659-70. https://doi.org/10.1093/fampra/cms029.

79. Gupta K. Addressing antibiotic resistance. Dis Mon. 2003;49:99 110. https://doi.org/10.1067/mda.2003.10.

80. Nicolle L, Anderson PAM, Conly J, Mainprize TC, Meuser J, Nickel JC, et al. Uncomplicated urinary tract infection in women. Current practice and the effect of antibiotic resistance on empiric treatment. Can Fam Physician. 2006;52:612-8.

81. Cohn EB, Schaeffer AJ. Urinary tract infections in adults. ScientificWorldJournal. 2004;4(Suppl 1):76-88. https://doi.org/ 10.1100/tsw.2004.50.

82. File TM, Tan JS. Urinary tract infections in the elderly. Geriatrics. 1989;44(Suppl A):15-9.

83. Das R, Perrelli E, Towle V, Van Ness PH, Juthani-Mehta M. Antimicrobial susceptibility of bacteria isolated from urine samples obtained from nursing home residents. Infect Control Hosp Epidemiol. 2009;30:1116-9. https://doi.org/10.1086/647981.

84. Gupta K, Hooton TM, Naber KG, Wullt B, Colgan R, Miller LG, et al. International clinical practice guidelines for the treatment of acute uncomplicated cystitis and pyelonephritis in women: A 2010 update by the Infectious Diseases Society of America and the European Society for Microbiology and Infectious Diseases. 
Clin Infect Dis. 2011;52:e103-20. https://doi.org/10.1093/cid/ ciq257.

85. Bradley MS, Beigi RH, Shepherd JP. A cost-minimization analysis of treatment options for postmenopausal women with dysuria. Am J Obstet Gynecol. 2019;221:505.e1-7. https://doi.org/10. 1016/j.ajog.2019.04.031.

86. Rothberg MB, Wong JB. All dysuria is local. A cost-effectiveness model for designing site-specific management algorithms. J Gen Intern Med. 2004;19:433-43. https://doi.org/10.1111/j.15251497.2004.10440.x.

87. Fenwick EA, Briggs AH, Hawke CI. Management of urinary tract infection in general practice: a cost-effectiveness analysis. $\mathrm{Br} \mathrm{J}$ Gen Pract. 2000;50:635-9.

88. McKinnell JA, Stollenwerk NS, Jung CW, Miller LG. Nitrofurantoin compares favorably to recommended agents as empirical treatment of uncomplicated urinary tract infections in a decision and cost analysis. Mayo Clin Proc. 2011;86:480-8. https://doi.org/10.4065/mcp.2010.0800.

89. Concia E, Bragantini D, Mazzaferri F. Clinical evaluation of guidelines and therapeutic approaches in multi drug-resistant urinary tract infections. J Chemother. 2017;29:19-28. https://doi.org/ 10.1080/1120009X.2017.1380397.

90. Faro S, Fenner DE. Urinary tract infections. Clin Obstet Gynecol. 1998;41:744-54. https://doi.org/10.1097/00003081-19980900000030 .

91. Nicolle LE. Update in adult urinary tract infection. Curr Infect Dis Rep. 2011;13:552-60. https://doi.org/10.1007/s11908-011-0212$\mathrm{x}$.

92. Naber KG. Which fluoroquinolones are suitable for the treatment of urinary tract infections? Int J Antimicrob Agents. 2001;17:33141.

93. Buckley BS, Lapitan MCM (2010) Drugs for treatment of urinary retention after surgery in adults. Cochrane Database Syst Rev CD008023. https://doi.org/10.1002/14651858.CD008023.pub2.

94. Burgio KL. Behavioral treatment of urinary incontinence, voiding dysfunction, and overactive bladder. Obstet Gynecol Clin N Am. 2009;36:475-91. https://doi.org/10.1016/j.ogc.2009.08.005.

95. Dörflinger A, Monga A. Voiding dysfunction. Curr Opin Obstet Gynecol. 2001;13:507-12. https://doi.org/10.1097/00001703200110000-00010.

96. Lavelle ES, Alam P, Meister M, Florian-Rodriguez M, ElmerLyon C, Kowalski J, et al. Antibiotic prophylaxis during catheter-managed postoperative urinary retention after pelvic reconstructive surgery: A randomized controlled trial. Obstet Gynecol. 2019;134:727-35. https://doi.org/10.1097/AOG. 0000000000003462.

97. Lusardi G, Lipp A, Shaw C (2013) Antibiotic prophylaxis for short-term catheter bladder drainage in adults. Cochrane Database Syst Rev CD005428. https://doi.org/10.1002/ 14651858.CD005428.pub2.

98. Newman DK, Willson MM. Review of intermittent catheterization and current best practices. Urol Nurs. 2011;31(12-28):48 quiz 29.

99. Ramsey S, Palmer M. The management of female urinary retention. Int Urol Nephrol. 2006;38:533-5. https://doi.org/10.1007/ s11255-005-5790-9.

100. Shokrpour M, Shakiba E, Sirous A, Kamali A. Evaluation the efficacy of prophylactic tamsulosin in preventing acute urinary retention and other obstructive urinary symptoms following colporrhaphy surgery. J Family Med Prim Care. 2019;8:722-7. https://doi.org/10.4103/jfmpc.jfmpc_18_19.

101. Sutkin G, Lowder JL, Smith KJ. Prophylactic antibiotics to prevent urinary tract infection during clean intermittent selfcatheterization (CISC) for management of voiding dysfunction after prolapse and incontinence surgery: a decision analysis. Int Urogynecol J Pelvic Floor Dysfunct. 2009;20:933-8. https://doi. org/10.1007/s00192-009-0885-y.
102. Tunitsky-Bitton E, Murphy A, Barber MD, Goldman HB, Vasavada S, Jelovsek JE. Assessment of voiding after sling: a randomized trial of 2 methods of postoperative catheter management after midurethral sling surgery for stress urinary incontinence in women. Am J Obstet Gynecol. 2015;212:597.e1-9. https://doi.org/10.1016/j.ajog.2014.11.033.

103. Dieter AA, Wu JM, Gage JL, Feliciano KM, Willis-Gray MG. Catheter burden following urogynecologic surgery. Am J Obstet Gynecol. 2019;221:507.e1-7. https://doi.org/10.1016/j.ajog.2019. 05.014 .

104. Hakvoort RA, Nieuwkerk PT, Burger MP, Emanuel MH, Roovers JP. Patient preferences for clean intermittent catheterisation and transurethral indwelling catheterisation for treatment of abnormal post-void residual bladder volume after vaginal prolapse surgery. BJOG. 2011;118:1324-8. https://doi.org/10.1111/j.1471-0528. 2011.03056.x.

105. Hentzen C, Haddad R, Ismael SS, Peyronnet B, Gamé X, Denys P, et al. Intermittent self-catheterization in older adults: predictors of success for technique learning. Int Neurourol J. 2018;22:65-71. https://doi.org/10.5213/inj.1835008.504.

106. Hentzen C, Haddad R, Ismael SS, Peyronnet B, Gamé X, Denys P, et al. Predictive factors of adherence to urinary self-catheterization in older adults. Neurourol Urodyn. 2019;38:770-8. https://doi.org/ 10.1002/nau.23915.

107. Kessler TM, Ryu G, Burkhard FC. Clean intermittent self-catheterization: a burden for the patient? Neurourol Urodyn. 2009;28: 18-21. https://doi.org/10.1002/nau.20610.

108. Meekins AR, Siddiqui NY, Amundsen CL, Kuchibhatla M, Dieter AA. Improving postoperative efficiency: an algorithm for expedited void trials after urogynecologic surgery. South Med J. 2017;110:785-90. https://doi.org/10.14423/SMJ. 0000000000000733 .

109. Mulder FEM, Hakvoort RA, de Bruin JP, van der Post JAM, Roovers J-PWR. Comparison of clean intermittent and transurethral indwelling catheterization for the treatment of overt urinary retention after vaginal delivery: a multicentre randomized controlled clinical trial. Int Urogynecol J. 2018;29:1281-7. https:// doi.org/10.1007/s00192-017-3452-y.

110. Sassani JC, Stork A, Ruppert K, Bradley MS. Variables associated with an inability to learn clean intermittent self-catheterization after urogynecologic surgery. Int Urogynecol J. 2019. https://doi. org/10.1007/s00192-019-03974-1.

111. Stoffel JT, Peterson AC, Sandhu JS, Suskind AM, Wei JT, Lightner DJ. AUA white paper on nonneurogenic chronic urinary retention: consensus definition, treatment algorithm, and outcome end points. J Urol. 2017;198:153-60. https://doi.org/10.1016/j. juro.2017.01.075.

112. Willis-Gray MG, Wu JM, Field C, Pulliam S, Husk KE, Brueseke $\mathrm{TJ}$, et al. Is a postvoid residual necessary? A randomized trial of two postoperative voiding protocols. Female Pelvic Med Reconstr Surg. 2019. https://doi.org/10.1097/SPV.0000000000000743.

113. Alas A, Hidalgo R, Espaillat L, Devakumar H, Davila GW, Hurtado E. Does spinal anesthesia lead to postoperative urinary retention in same-day urogynecology surgery? A retrospective review. Int Urogynecol J. 2019;30:1283-9. https://doi.org/10. 1007/s00192-019-03893-1.

114. Shatkin-Margolis A, Yook E, Hill AM, Crisp CC, Yeung J, Kleeman S, et al. Self-removal of a urinary catheter after Urogynecologic surgery: A randomized controlled trial. Obstet Gynecol. 2019;134:1027-36. https://doi.org/10.1097/AOG. 0000000000003531.

115. Hooton TM, Bradley SF, Cardenas DD, Colgan R, Geerlings SE, Rice JC, et al. Diagnosis, prevention, and treatment of catheterassociated urinary tract infection in adults: 2009 international clinical practice guidelines from the Infectious Diseases Society of 
America. Clin Infect Dis. 2010;50:625-63. https://doi.org/10. 1086/650482.

116. Madersbacher H, Cardozo L, Chapple C, Abrams P, ToozsHobson P, Young JS, et al. What are the causes and consequences of bladder overdistension? ICI-RS 2011. Neurourol Urodyn. 2012;31:317-21. https://doi.org/10.1002/nau.22224.

117. Society of Gynecologic Surgeons A Guide to Female Clean Intermitent Self Catheterization. https://vimeo.com/261183016. Accessed 4 Apr 2020.

118. Balk EM, Rofeberg VN, Adam GP, Kimmel HJ, Trikalinos TA, Jeppson PC. Pharmacologic and nonpharmacologic treatments for urinary incontinence in women: A systematic review and network meta-analysis of clinical outcomes. Ann Intern Med. 2019;170: 465-79. https://doi.org/10.7326/M18-3227.

119. Simpson AN, Garbens A, Dossa F, Coyte PC, Baxter NN, McDermott CD. A cost-utility analysis of nonsurgical treatments for stress urinary incontinence in women. Female Pelvic Med Reconstr Surg. 2019;25:49-55. https://doi.org/10.1097/SPV. 0000000000000502 .

120. Richardson K, Fox C, Maidment I, Steel N, Loke YK, Arthur A, et al. Anticholinergic drugs and risk of dementia: case-control study. BMJ. 2018;361:k1315. https://doi.org/10.1136/bmj.k1315.

121. Kelleher C, Hakimi Z, Zur R, Siddiqui E, Maman K, Aballéa S, et al. Efficacy and tolerability of Mirabegron compared with Antimuscarinic monotherapy or combination therapies for overactive bladder: A systematic review and network meta-analysis. Eur Urol. 2018;74:324-33. https://doi.org/10.1016/j.eururo.2018.03. 020 .

122. Chen H-L, Chen T-C, Chang H-M, Juan Y-S, Huang W-H, Pan H$\mathrm{F}$, et al. Mirabegron is alternative to antimuscarinic agents for overactive bladder without higher risk in hypertension: a systematic review and meta-analysis. World J Urol. 2018;36:1285-97. https://doi.org/10.1007/s00345-018-2268-9.

123. Barnes KL, Dunivan G, Jaramillo-Huff A, Krantz T, Thompson J, Jeppson P. Evaluation of smartphone pelvic floor exercise applications using standardized scoring system. Female Pelvic Med Reconstr Surg. 2019;25:328-35. https://doi.org/10.1097/SPV. 0000000000000563.

124. Nekkanti S, Wu JM, Hudson CO, Pandya LK, Dieter AA. A randomized trial comparing continence pessary to a disposable intravaginal device [poise impressa ${ }^{\circledR}$ ] for the non-surgical management of stress urinary incontinence. Female Pelvic Med Reconstr Surg. 2019;26:S95-6.

125. Balk E, Adam GP, Kimmel H, Rofeberg V, Saeed I, Jeppson P, Trikalinos T (2018) Nonsurgical treatments for urinary incontinence in women: A systematic review update. Agency for Healthcare Research and Quality (US), Rockville (MD).

126. Wald A, Bharucha AE, Cosman BC, Whitehead WE. ACG clinical guideline: management of benign anorectal disorders. Am J Gastroenterol. 2014;109:1141-57; (Quiz) 1058. https://doi.org/ 10.1038/ajg.2014.190.

127. Paquette IM, Varma MG, Kaiser AM, Steele SR, Rafferty JF. The american society of colon and rectal surgeons' clinical practice guideline for the treatment of fecal incontinence. Dis Colon Rectum. 2015;58:623-36. https://doi.org/10.1097/DCR. 0000000000000397 .

128. Ridgeway BM, Weinstein MM, Tunitsky-Bitton E. American Urogynecologic Society (AUGS) best-practice statement on evaluation of obstructed defecation. Female Pelvic Med Reconstr Surg. 2018;24:383-91. https://doi.org/10.1097/SPV. 0000000000000635 .

129. American Urogynecologic Society (AUGS) Guidelines and Statements Committee, Carberry CL, Tulikangas PK, Ridgeway BM, Collins SA, Adam RA. American Urogynecologic Society (AUGS) best practice statement: evaluation and counseling of patients with pelvic organ prolapse. Female Pelvic Med
Reconstr Surg. 2017;23:281-7. https://doi.org/10.1097/SPV. 0000000000000424.

130. Bradley CS, Zimmerman MB, Qi Y, Nygaard IE. Natural history of pelvic organ prolapse in postmenopausal women. Obstet Gynecol. 2007;109:848-54. https://doi.org/10.1097/01.AOG. 0000255977.91296.5d.

131. Gilchrist AS, Campbell W, Steele H, Brazell H, Foote J, Swift S. Outcomes of observation as therapy for pelvic organ prolapse: a study in the natural history of pelvic organ prolapse. Neurourol Urodyn. 2013;32:383-6. https://doi.org/10.1002/nau.22298.

132. Dumoulin C, Hunter KF, Moore K, Bradley CS, Burgio KL, Hagen $S$, et al. Conservative management for female urinary incontinence and pelvic organ prolapse review 2013: summary of the 5th international consultation on incontinence. Neurourol Urodyn. 2016;35:15-20. https://doi.org/10.1002/nau.22677.

133. Braekken IH, Majida M, Engh ME, Bø K. Can pelvic floor muscle training reverse pelvic organ prolapse and reduce prolapse symptoms? An assessor-blinded, randomized, controlled trial. Am J Obstet Gynecol. 2010;203:170.e1-7. https://doi.org/10.1016/j. ajog.2010.02.037.

134. Modi RM, Hinton A, Pinkhas D, Groce R, Meyer MM, Balasubramanian $\mathrm{G}$, et al. Implementation of a defecation posture modification device: impact on bowel movement patterns in healthy subjects. J Clin Gastroenterol. 2019;53:216-9. https:/ doi.org/10.1097/MCG.0000000000001143.

135. ACOOAGCOPB, Dunivan GC, Chen CCG, Rogers RG (2019) ACOG practice bulletin no. 210: fecal incontinence. Obstet Gynecol 133:e260-e273. https://doi.org/10.1097/AOG. 0000000000003187.

136. Forte ML, Andrade KE, Butler M, Lowry AC, Bliss DZ, Slavin JL, Kane RL (2016) Treatments for Fecal Incontinence. Agency for Healthcare Research and Quality.

137. Deutekom M, Dobben A (2005) Plugs for containing faecal incontinence. Cochrane Database Syst Rev CD005086. https://doi. org/10.1002/14651858.CD005086.pub2.

138. Chen N, Zhou M, Dong X, Qu J, Gong F, Han Y, et al. Epidemiological and clinical characteristics of 99 cases of 2019 novel coronavirus pneumonia in Wuhan, China: a descriptive study. Lancet. 2020;395:507-13. https://doi.org/10.1016/S01406736(20)30211-7.

139. American College of Surgeons COVID-19: Recommendations for Management of Elective Surgical Procedures. https://www.facs. org/covid-19/clinical-guidance/elective-surgery. Accessed 2 Apr 2020.

140. Weber Lebrun EE, Moawad NS, Rosenberg EI, Morey TE, Davies L, Collins WO, et al. COVID-19 pandemic: staged Management of Surgical Services for gynecology and obstetrics. Am J Obstet Gynecol. 2020. https://doi.org/10.1016/j.ajog.2020.03.038.

141. Society of American Gastrointestinal and Endoscopic Surgeons SAGES and EAES Recommendations Regarding Surgical Response to COVID-19 Crisis. https://www.sages.org/ recommendations-surgical-response-covid-19/. Accessed 2 Apr 2020.

142. Chen X, Liu Y, Gong Y, Guo X, Zuo M, Li J, et al. Perioperative Management of Patients Infected with the novel coronavirus: recommendation from the joint task force of the Chinese Society of Anesthesiology and the Chinese Association of Anesthesiologists. Anesthesiology. 2020. https://doi.org/10.1097/ALN. 0000000000003301.

143. Bacheller CD, Bernstein JM. Urinary tract infections. Med Clin North Am. 1997;81:719-30. https://doi.org/10.1016/s00257125(05)70542-3.

Publisher's note Springer Nature remains neutral with regard to jurisdictional claims in published maps and institutional affiliations. 